Research Article

\title{
Parametric Vibration Analysis of a Six-Degree-of-Freedom Electro-Hydraulic Stewart Platform
}

\author{
Xiaoming Yuan $(\mathbb{D}$, Yue Tang $\mathbb{D}$, Weiqi Wang, and Lijie Zhang $(\mathbb{D}$ \\ Hebei Provincial Key Laboratory of Heavy Machinery Fluid Power Transmission and Control, Yanshan University, \\ Qinhuangdao 066004, China
}

Correspondence should be addressed to Xiaoming Yuan; yuanxiaoming@ysu.edu.cn

Received 16 March 2021; Revised 1 July 2021; Accepted 30 July 2021; Published 12 August 2021

Academic Editor: Ling Zhou

Copyright (c) 2021 Xiaoming Yuan et al. This is an open access article distributed under the Creative Commons Attribution License, which permits unrestricted use, distribution, and reproduction in any medium, provided the original work is properly cited.

\begin{abstract}
Electro-hydraulic Stewart 6-DOF platform is a 6-DOF parallel mechanism combined with the electro-hydraulic servo control system, which is widely used in the field of construction machinery. In actual working conditions, the flow and pressure pulsation of the hydraulic oil output from the hydraulic leg of the electro-hydraulic Stewart platform are inevitable, so the equivalent stiffness of the platform leg will change, and the stiffness parameters of the transmission system will change, resulting in vibration, which will affect the accuracy of the platform. This paper considering the fluid unit equivalent stiffness cyclical fluctuations and leg, on the basis of the relationship between hydraulic stiffness, constructs the electric hydraulic Stewart platform machine vibration dynamics equation, fluid coupling parameters of vibration parameters using the method of the multiscale approximate analytic formula of the main resonance and combination resonance are derived, and the system parameters vibration time-domain response and frequency response under two different poses are discussed. Results show that the system first to six order natural frequency and the first to the sixth order natural frequency and frequency of hydraulic oil equivalent stiffness of the combination of frequency will have an effect on the parameters of the system vibration. In the main resonance, the dominant frequency is mainly the first to sixth order natural frequency of the system; in the combined resonance, the dominant frequency is the combined frequency. Through the parameter vibration analysis of two different positions of the platform, it is concluded that when the platform is in an asymmetric position, each leg of the system is more involved in vibration. This study can provide the reference for the subsequent dynamic optimization and reliability analysis of the electro-hydraulic Stewart platform.
\end{abstract}

\section{Introduction}

The Stewart 6-DOF platform is a typical parallel mechanism, which connects the base and the moving platform through six kinematic chains and controls the movement of the platform. Because its design and research involve a series of high and new technology fields such as machinery [1], hydraulic [2-4], control [5], computer, signal [6], and sensor [7], it has integrated the knowledge of multiple disciplines such as electromechanical and hydraulic and has been attached great importance by the academic circles [8]. In addition, compared with the series mechanism, the platform has the advantages of stable structure and high precision [9] and is widely applied to machine tools [10], vehicles [11], medical equipment [12], sensors [13], spacecraft [14, 15], telescopes [16], and other fields. Therefore, the Stewart platform also has a strong attraction to the industry. The platform becomes an electro-hydraulic Stewart platform when the hydraulic legs of the Stewart platform are incorporated into the electro-hydraulic servo control system. The electro-hydraulic platform has strong coupling; the platform mass and the equivalent stiffness of the leg will affect the dynamic characteristics of the platform [17]. When the legs are driven by electro-hydraulic servo, the controllability of the platform can be increased. However, compared with the mechanical leg, the equivalent stiffness of the hydraulic leg oil is lower, which leads to the change of the dynamic characteristics of the platform [18-20]. 
Under the condition that the external excitation frequency approaches to the natural frequency of the platform, vibration and failure will be caused [21, 22]. Therefore, the analysis of platform dynamics is of great significance.

In real conditions, the pressure pulsation of the hydraulic oil output by the hydraulic cylinder on the leg of the electro-hydraulic Stewart platform is inevitable, so the equivalent stiffness of the hydraulic oil will change, and the stiffness parameters of the transmission system will change, leading to the generation of vibration [23]. The vibration induced by the time-varying parameters (stiffness, damping, mass, or moment of mass inertia) of the system is called parametric vibration. The time-varying excitation appears in the system differential equation as an inhomogeneous term, which is called the external excitation. When it appears as a coefficient in the equation of motion, it is called a parametric excitation. The main resonance occurs when the natural frequency of the system is close to the excitation frequency of the external excitation. When the natural frequency of the system is close to the sum of the excitation frequency of the external excitation and the pulsation frequency of the equivalent stiffness of the fluid, the combined resonance phenomenon occurs. With the development of science, equipment in various fields have higher requirements for operating accuracy, so it is necessary to reduce the negative impact of vibration on equipment accuracy [24,25]. Therefore, it is of great significance to study the problem of parametric vibration to reduce system vibration.

For parametric vibration problems, scholars mainly use the numerical method and analytical method to solve them, among which the analytical method includes the L-P method [26] (Lindstedt-Poincare method), multiscale method [27], and many other approximate analytical solutions. The multiscale method is an improved approximate analytical method for nonlinear differential equations based on the average method. For the solution of different problems, the multiscale method has a high versatility, so it has been widely used in recent years $[28,29]$. He et al. adopted the multiscale method and obtained the nonlinear natural frequency, amplitudefrequency equation, and time history of the beam under the hinged boundary condition by Galerkin approximation discretization [30]. Chen et al. studied the transverse nonlinear vibration of axially accelerated viscoelastic beams with principal parameter resonance and applied the multiscale method to the governing equation to determine the steady-state response [31].

This paper considering the hydraulic oil equivalent stiffness cyclical fluctuations and leg, on the basis of relationship between hydraulic stiffness, constructs the electric hydraulic Stewart platform machine vibration dynamics equation, fluid coupling parameters of vibration parameters using the method of the multiscale approximate analytic formula of the primary resonance and combination resonance are derived, and the system parameters vibration time-domain response and frequency response under two different poses are discussed.

\section{Dynamic Modeling of Electro-Hydraulic Stewart Platform}

2.1. Position Analysis of Electro-Hydraulic Platform. The geometrical structure diagram of the electro-hydraulic Stewart 6-DOF platform is shown in Figure 1. The electrohydraulic Stewart 6-DOF platform consists of three parts: a top platform, a base platform, and six legs of the same size. Among them, the top platform is a moving platform, and the base platform is a fixed platform. The six supporting legs are connected with the upper and base platforms, respectively, through ball hinges and universal joints. The six legs are composed of hydraulic cylinders and sensors that provide input excitation to the legs to ensure movement of the platform.

The motional coordinate system A-XYZ is established at the nature heart of the top platform, while the static one $\mathrm{B}-x y z$ is established at the nature heart of the base platform. $A$ and $B$ are the nature heart of the top platform and the base one, respectively. The distribution of six balls hinged on the top platform is determined by using $a_{i}$ $(i=1,2, \ldots, 6)$, and the six universal joints on the base platform are $b_{i}(i=1,2, \ldots, 6)$. By using $\mathbf{R}_{a}$ and $\mathbf{E}$, the rotation matrix and displacement matrix of the motional coordinate system relative to the static one can be expressed. We assumed that $m$ is the mass of the top platform, while $I_{x}, I_{y}$, and $I_{z}$ are, respectively, the rotation inertia of the top platform about the $X Y Z$ axis.

By using $P_{x}, P_{y}$, and $P_{z}$, the movement of the moving coordinate system $\mathrm{A}-X Y Z$ in three directions relative to the absolute static coordinate system B- $x y z$ can be represented. By using $\alpha, \beta$, and $\gamma$, the rotation in three directions of the moving coordinate system $\mathrm{A}-X Y Z$ with respect to the absolute static coordinate system B- $x y z$ can be expressed. The above six variables determine the position of the platform.

Therefore, the expression of the rotation matrix $\mathbf{R}_{a}$ and displacement matrix $\mathbf{E}$ is as follows:

$$
\begin{aligned}
\mathbf{R}_{a} & =\left(\begin{array}{ccc}
c \gamma c \beta & c \gamma s \beta s \alpha-s \gamma c \alpha & s \gamma s \alpha+c \gamma s \beta c \alpha \\
s \gamma c \beta & c \gamma c \alpha+s \gamma s \beta s \alpha & s \gamma s \beta c \alpha-c \gamma c \alpha \\
-s \beta & c \beta s \alpha & c \beta c \alpha
\end{array}\right), \\
\mathbf{E} & =\left(\begin{array}{lll}
P_{x} & P_{y} & P_{z}
\end{array}\right)^{T},
\end{aligned}
$$

where $s \alpha=\sin \alpha$ and $c \alpha=\cos \alpha$.

The expression of the six leg positions is as follows:

$$
\mathbf{l}_{i}=\mathbf{R}_{a} \times \mathbf{A}_{i}+\mathbf{E}-\mathbf{a}_{i},
$$

where $i=1,2, \ldots, 6$.

In this paper, the two poses' parameter vibration is analyzed. The parameters of the two poses are as follows:

Pose 1:

$$
\begin{aligned}
\mathbf{x} & =\left(P_{x}, P_{y}, P_{z}, \alpha, \beta, \gamma\right)^{T} \\
& =\left(\begin{array}{llllll}
0 & 0 & 2 \sqrt{3} & 0 & 0 & 0
\end{array}\right)^{T} .
\end{aligned}
$$

Pose 2: 


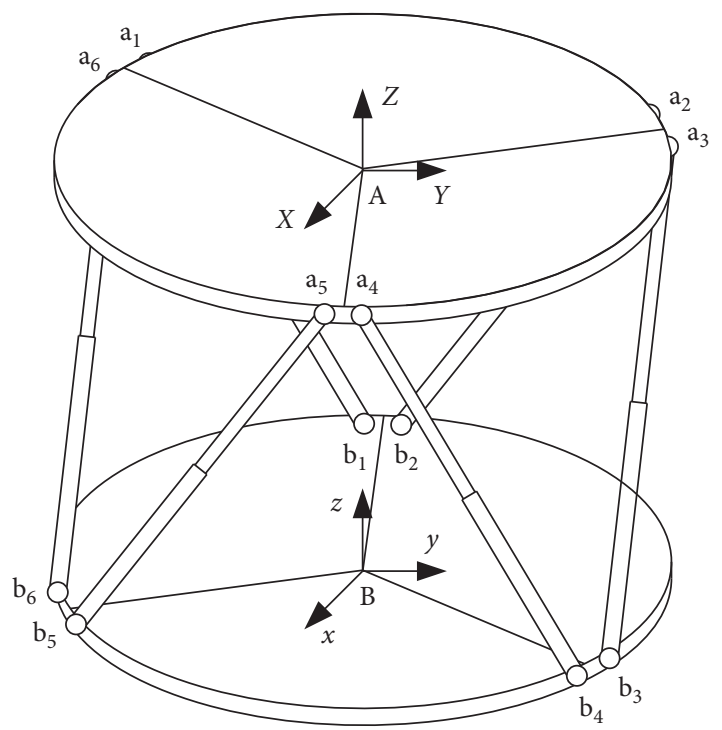

FIgURE 1: Schematic diagram of the 6-DOF platform structure.

$$
\begin{aligned}
\mathbf{x} & =\left(P_{x}, P_{y}, P_{z}, \alpha, \beta, \gamma\right)^{T} \\
& =\left(\begin{array}{llllll}
1 & 1 & 2 \sqrt{3} & 5 & 5 & 10
\end{array}\right)^{T} .
\end{aligned}
$$

The initial position of the platform which is shown in Figure 1 is represented by pose 1 , and it is symmetrical. Furthermore, the general position of the platform, which has no symmetry, is represented by pose 2 , and the position parameters are all changed. Through the analysis of the dynamic characteristics of different postures, whether the change of the platform position has an effect on its parameter vibration is judged.

Physical parameters of the six-degree-of-freedom electro-hydraulic platform are shown in Table 1:

\subsection{Dynamic Equation of Electro-Hydraulic Stewart 6-DOF} Platform Transmission System. The dynamic model of the electro-hydraulic platform established in this paper adopts the following assumptions.

The dynamic model of the electro-hydraulic Stewart platform adopts the following assumptions:

(1) The components of the Stewart platform are all rigid bodies, that is, the elastic deformation of other components is not considered

(2) In the machine construction of the platform, the supporting legs work as a linear spring along the longitudinal direction of the legs

(3) The leg mass is much smaller than the top platform mass, so the effect caused by the leg mass would become negligible

(4) The friction which is caused by the moving components can be neglected

(5) Ignore the processing error and assembly error of each component
2.2.1. Velocity Analysis and Jacobian Matrix of Electro-Hydraulic Platform. Set the generalized coordinates of the platform to

$$
\mathbf{x}=\left(P_{x}, P_{y}, P_{z}, \alpha, \beta, \gamma\right)^{T}
$$

Thus, the velocity $\mathbf{V}$ and angular velocity $\boldsymbol{\omega}$ of the moving platform can be expressed as

$$
\begin{aligned}
& \mathbf{V}=\dot{P}=\left(\begin{array}{lll}
\dot{P}_{x} & \dot{P}_{y} & \dot{P}_{z}
\end{array}\right), \\
& \boldsymbol{\omega}=\dot{\theta}=(\dot{\alpha}, \dot{\beta}, \dot{\gamma}) .
\end{aligned}
$$

By assuming that $\mathbf{r}$ is the vector diameter of spherical hinge points relative to the nature heart of the top platform, $V_{\mathrm{S}}$ is the speed of ball hinge points, $\mathbf{e}$ is the unit direction vector of the leg, and $v$ is the change rate of the length of the leg rod; thus, the expression of them is as follows:

$$
\begin{aligned}
\mathbf{r}_{i} & =\mathbf{R}_{a} \mathbf{A}_{i}, \\
\mathbf{V}_{a i} & =\mathbf{V}+\boldsymbol{\omega} \times \mathbf{r}_{i}, \\
v_{i} & =V_{a i} \cdot e_{i},
\end{aligned}
$$

where $i=1,2, \ldots, 6$.

Substituting (8) into (9), we can obtain

$$
\mathbf{v}_{i}=\left[\begin{array}{ll}
e_{i}^{T} & \left(r_{i} \times e_{i}\right)^{T}
\end{array}\right]\left[\begin{array}{c}
V \\
\boldsymbol{\omega}
\end{array}\right] .
$$

Then, the speed of the six legs is

$$
\left[\begin{array}{c}
v_{1} \\
v_{2} \\
v_{3} \\
v_{4} \\
v_{5} \\
v_{6}
\end{array}\right]=\left[\begin{array}{cc}
e_{1}^{T} & \left(r_{1} \times e_{1}\right)^{T} \\
e_{2}^{T} & \left(r_{2} \times e_{2}\right)^{T} \\
e_{3}^{T} & \left(r_{3} \times e_{3}\right)^{T} \\
e_{4}^{T} & \left(r_{4} \times e_{4}\right)^{T} \\
e_{5}^{T} & \left(r_{5} \times e_{5}\right)^{T} \\
e_{6}^{T} & \left(r_{6} \times e_{6}\right)^{T}
\end{array}\right]\left[\begin{array}{c}
V \\
\boldsymbol{\omega}
\end{array}\right] .
$$

The shorthand for which is

$$
[v]=[J]\left[\begin{array}{ll}
V & \boldsymbol{\omega}
\end{array}\right]^{T},
$$

where $[J]$ is the Jacobian matrix.

\subsubsection{Dynamic Equation for Constructing Electro-Hydraulic} Platform. The equivalent stiffness of oil in the six-leg hydraulic cylinder is determined by $k_{i}(i=1,2, \ldots, 6)$, damping is expressed by $c_{i}(i=1,2, \ldots, 6)$, and output force of the leg hydraulic cylinder is $f_{a i}(i=1,2, \ldots, 6)$.

The inertial force and its virtual power received by the platform are sorted and calculated, and according to the principle of virtual power, the following can be obtained:

$$
\begin{aligned}
\boldsymbol{\delta} p= & \left(f_{a}-k \Delta l-c l\right) \cdot \boldsymbol{\delta} \dot{l}+(-m \ddot{p}) \cdot \boldsymbol{\delta} \dot{p} \\
& +\left(-I_{a} \dot{\boldsymbol{\omega}}-\boldsymbol{\omega} \times I_{a} \boldsymbol{\omega}\right) \cdot \boldsymbol{\delta} \boldsymbol{\omega}=0 .
\end{aligned}
$$


TABLE 1: Physical parameters of the electro-hydraulic Stewart platform (Unit: Si).

\begin{tabular}{|c|c|c|}
\hline Name & Symbol & Value \\
\hline Top platform radius & $R$ & 2 \\
\hline Thickness of top platform & $D$ & 0.2 \\
\hline Quality of top platform & $m$ & 20 \\
\hline Inertia of the top platform about the $X$ axis & $I_{x}$ & 20.07 \\
\hline Inertia of the top platform about the $Y$ axis & $I_{y}$ & 20.07 \\
\hline Inertia of top platform about the $Z$ axis & $I_{z}$ & 40 \\
\hline Universal joint at position 1 & $\mathbf{b}_{1}$ & {$\left[\begin{array}{lll}(1 / 2) R & (\sqrt{3} / 2) R & 0\end{array}\right]_{T}^{T}$} \\
\hline Universal joint at position 2 & $\mathbf{b}_{2}$ & {$\left[\begin{array}{lll}(1 / 2) R & (\sqrt{3} / 2) R & 0\end{array}\right]^{T}$} \\
\hline Universal joint at position 3 & $\mathbf{b}_{3}$ & $(1 / 2) R-(\sqrt{3} / 2) R \quad 0]_{T}^{T}$ \\
\hline Universal joint at position 4 & $\mathbf{b}_{4}$ & $(1 / 2) R-(\sqrt{3} / 2) R \quad 0]^{T}$ \\
\hline Universal joint at position 5 & $\mathbf{b}_{5}$ & {$\left[\begin{array}{lll}-R & 0 & 0\end{array}\right]^{T}$} \\
\hline Universal joint at position 6 & $\mathbf{b}_{6}$ & {$\left[\begin{array}{lll}-R & 0 & 0\end{array}\right]^{T}$} \\
\hline Ball hinge position 1 & $\mathbf{a}_{1}$ & {$\left[\begin{array}{lll}-(1 / 2) R & (\sqrt{3} / 2) R & 0\end{array}\right]^{T}$} \\
\hline Ball hinge position 2 & $\mathbf{a}_{2}$ & {$\left[\begin{array}{lll}R & 0 & 0\end{array}\right]_{T}^{T}$} \\
\hline Ball hinge position 3 & $\mathbf{a}_{3}$ & {$\left[\begin{array}{lll}R & 0 & 0\end{array}\right]^{T}$} \\
\hline Ball hinge position 4 & $\mathbf{a}_{4}$ & {$\left[\begin{array}{lll}-(1 / 2) R-(\sqrt{3} / 2) R & 0\end{array}\right]^{T}$} \\
\hline Ball hinge position 5 & $\mathbf{a}_{5}$ & {$\left[\begin{array}{lll}-(1 / 2) R & -(\sqrt{3} / 2) R & 0\end{array}\right]^{T}$} \\
\hline Ball hinge position 6 & $\mathbf{a}_{6}$ & {$\left[\begin{array}{lll}-(1 / 2) R & (\sqrt{3} / 2) R & 0\end{array}\right]^{T}$} \\
\hline
\end{tabular}

The Coriolis force and centrifugal force on the platform are ignored. Equation (13) can be simplified to

$$
\boldsymbol{\delta} p=\left(f_{a}-k J x-c J \dot{x}\right)^{T} J \boldsymbol{\delta} \dot{x}-\left(\begin{array}{c}
m \ddot{p} \\
I_{a} \dot{\boldsymbol{\omega}}
\end{array}\right)^{T} \boldsymbol{\delta} \dot{x}=0 .
$$

In the formula above, $\dot{x}=(\dot{p} \omega)^{T}$. The components of $\delta \dot{x}$ have no effect on each other, so the coefficients are all zero; thus, the dynamic equation can be expressed as

$$
\mathbf{M} \ddot{x}+\mathbf{C} \dot{x}+\mathbf{K x}=\mathbf{J}^{T} \mathbf{f}_{a},
$$

where $\quad \mathbf{M}=\left[\begin{array}{cc}m I^{3 \times 3} & 0 \\ 0 & I_{a}\end{array}\right], \quad \mathbf{K}=J^{T} k J, \quad \mathbf{C}=J^{T} c J, \quad \mathbf{k}=$ $\operatorname{diag}\left(\left[\begin{array}{llllll}k_{1} & k_{2} & k_{3} & k_{4} & k_{5} & k_{6}\end{array}\right]\right)$,

$\mathbf{c}=\operatorname{diag}\left(\left[\begin{array}{llllll}c_{1} & c_{2} & c_{3} & c_{4} & c_{5} & c_{6}\end{array}\right]\right)$,

$\mathbf{f}_{a}=\left(\begin{array}{llllll}f_{a 1} & f_{a 2} & f_{a 3} & f_{a 4} & f_{a 5} & f_{a 6}\end{array}\right)^{T}$.

and

\section{Parametric Vibration Equation of Electro- Hydraulic Stewart 6-DOF Platform Transmission System}

3.1. Time-Varying Equivalent Stiffness of Gas-Liquid Mixture. Under actual working conditions, the pressure fluctuation of the hydraulic oil output by the hydraulic cylinder on the leg of the electro-hydraulic Stewart platform is inevitable; thus, the hydraulic oil equivalent stiffness will change. Assuming that the dynamic pressure changes in accordance with cosine, then the expression of the instantaneous pressure pulsation can be obtained as

$$
\mathbf{p}=\bar{p}+\Delta p\left[e^{j \omega_{0} t}+e^{-j \omega_{0} t}\right],
$$

where $\bar{p}$ represents steady-state pressure $(\mathrm{Pa}), \Delta p$ represents pressure pulsation amplitude $(\mathrm{Pa})$, and $\omega_{o}$ represents pressure pulsation angular frequency $(\mathrm{rad} / \mathrm{s})$.

It is known that the hydraulic oil stiffness is a function of the hydraulic oil pressure and the initial gas content under isothermal conditions. Therefore, under a certain initial gas content, the equivalent stiffness fluctuation of hydraulic oil is similar to the pressure fluctuation of hydraulic oil and can be expressed as

$$
k_{f}=\overline{k_{f}}+k_{f v} \cos \omega_{e} t=\overline{k_{f}}\left[1+\varepsilon e^{j \omega_{e} t}+c c\right],
$$

where $\overline{k_{f}}$ represents steady-state fluid stiffness $(\mathrm{N} / \mathrm{m}), k_{f v}$ represents dynamic fluid stiffness amplitude $(\mathrm{N} / \mathrm{m}), \varepsilon$ represents small parameter, $\varepsilon=k_{f v} / 2 \overline{k_{f}}$, $\omega_{e}$ represents angular frequency of fluid stiffness fluctuation ( $\mathrm{rad} / \mathrm{s})$, and $c c$ represents the complex conjugate.

Fluid density is a function of fluid pressure, and the pressure fluctuation is bound to cause the change of fluid mass, but the change of fluid mass is very small under the steady condition. Therefore, parametric vibration analysis mainly studies the system parametric vibration response when the equivalent stiffness of fluid changes.

3.2. Construct Parametric Vibration Equations. Based on the lumped parameter method, the fluid pressure is uniformly distributed in the whole control body, and its variation law is consistent. Because of the pressure pulsation, the motion of the platform leg is affected by the dynamic pressure. Assuming that the dynamic pressure is in the law of cosine change and expressed as an exponential function, the system parameter vibration differential equation is shown as follows:

$$
\begin{aligned}
\delta p= & \left(\left(e^{j \omega_{o} t}+e^{-j \omega_{o} t}\right) F_{o}-\overline{k_{f}}\left(1+\varepsilon e^{j \omega_{e} t}+c c\right) J x-c J \dot{x}\right)^{T} J \delta \dot{x} \\
& -\left(\begin{array}{c}
m \ddot{p} \\
I_{a} \dot{\omega}
\end{array}\right)^{T} \delta \dot{x}=0,
\end{aligned}
$$

where $F_{o}$ represents the pressure pulsation amplitude matrix and $F_{0}=\left[\begin{array}{llllll}\Delta f_{a 1} & \Delta f_{a 2} & \Delta f_{a 3} & \Delta f_{a 4} & \Delta f_{a 5} & \Delta f_{a 6}\end{array}\right]^{T}$.

Equation (18) is expressed in the matrix form as

$$
\mathbf{m} \ddot{x}+\mathbf{c} \dot{x}+\mathbf{k} \mathbf{x}=\Delta \mathbf{F}-\Delta \mathbf{k} \mathbf{x},
$$


where $\mathbf{k}$ represents the steady-state stiffness matrix, $k=\overline{k_{f}} J^{T} J, \Delta \mathbf{F}$ represents the excitation force vector, $\Delta \mathbf{F}=$ $\left(e^{j \omega_{o} t}+e^{-j \omega_{o} t}\right) J^{T} F_{o}, \Delta \mathbf{k}$ represents the incremental stiffness matrix, and $\Delta \mathbf{k}=\overline{k_{\mathrm{f}}} \mathcal{E}\left(\mathrm{e}^{\mathrm{j} \omega_{\mathrm{e}} t}+\mathrm{e}^{-\mathrm{j} \omega_{\mathrm{e}} t}\right) J^{T} J$.

\section{Derivation of Approximate Analytical Formula for Parametric Vibration of Electro- Hydraulic Stewart Platform}

Through the relevant knowledge of vibration mechanics, the regular mode $\psi$ and spectral matrix $\Lambda$ of the system of the platform transmission system are solved, and equation (19) is regularized to obtain

$$
\ddot{\boldsymbol{\eta}}+C_{N} \dot{\boldsymbol{\eta}}+\Lambda \boldsymbol{\eta}=\Delta \mathbf{Q}-\Delta \mathbf{k}_{N} \boldsymbol{\eta},
$$

where $\eta$ represents the regular generalized displacement vector, $\eta=\left[\begin{array}{llllll}\eta_{1} & \eta_{2} & \eta_{3} & \eta_{4} & \eta_{5} & \eta_{6}\end{array}\right]^{T}, C_{N}$ represents the canonical damping matrix, $C_{N}=\left[\begin{array}{ccc}C_{N 11} & \cdots & C_{N 16} \\ \vdots & \ddots & \vdots \\ C_{N 61} & \cdots & C_{N 66}\end{array}\right], \Delta \mathbf{Q}$ represents the regularly excited force vector, $\Delta Q=\left(e^{j \omega_{o} t}+e^{-j \omega_{o} t}\right) \psi^{T} J^{T} F_{o}, \Delta \mathbf{k}_{N}$ represents the regular stiffness increment matrix, and $\Delta k_{N}=$ $\varepsilon\left(e^{j \omega_{e} t}+e^{-j \omega_{e} t}\right)\left[\begin{array}{ccc}\Delta k_{N 11} & \cdots & \Delta k_{N 16} \\ \vdots & \ddots & \vdots \\ \Delta k_{N 61} & \cdots & \Delta k_{N 66}\end{array}\right]$.

4.1. Approximate Analytical Derivation of Main Resonance of Transmission System of Electro-Hydraulic Platform. By using the multiscale method, the quadratic approximate solution, as shown in the following equation, is introduced:

$$
\eta_{i}(t)=\eta_{i 0}\left(T_{0}, T_{1}\right)+\varepsilon \eta_{i 1}\left(T_{0}, T_{1}\right),
$$

where $T_{0}=t$ and $T_{1}=\varepsilon t$.
For convenience of derivation, the following parameters are introduced into small parameters to obtain

$$
\left\{\begin{array}{l}
c_{N i j}=\varepsilon c_{N i j}^{\prime}, \\
F_{o}=\varepsilon F_{o}^{\prime} .
\end{array}\right.
$$

Substitute the above equation into equation (20), and using the same power coefficient equal, the equation of zero power term can be calculated as

$$
\left\{\begin{array}{l}
\frac{\mathrm{d}^{2} \eta_{10}}{\mathrm{~d} T_{0}^{2}}+\omega_{n 1}^{2} \eta_{10}=0 \\
\frac{\mathrm{d}^{2} \eta_{20}}{\mathrm{~d} T_{0}^{2}}+\omega_{n 2}^{2} \eta_{20}=0 \\
\frac{\mathrm{d}^{2} \eta_{30}}{\mathrm{~d} T_{0}^{2}}+\omega_{n 3}^{2} \eta_{30}=0 \\
\frac{\mathrm{d}^{2} \eta_{40}}{\mathrm{~d} T_{0}^{2}}+\omega_{n 4}^{2} \eta_{40}=0 \\
\frac{\mathrm{d}^{2} \eta_{50}}{\mathrm{~d} T_{0}^{2}}+\omega_{n 5}^{2} \eta_{50}=0 \\
\frac{\mathrm{d}^{2} \eta_{60}}{\mathrm{~d} T_{0}^{2}}+\omega_{n 6}^{2} \eta_{60}=0 .
\end{array}\right.
$$

The first power term is 


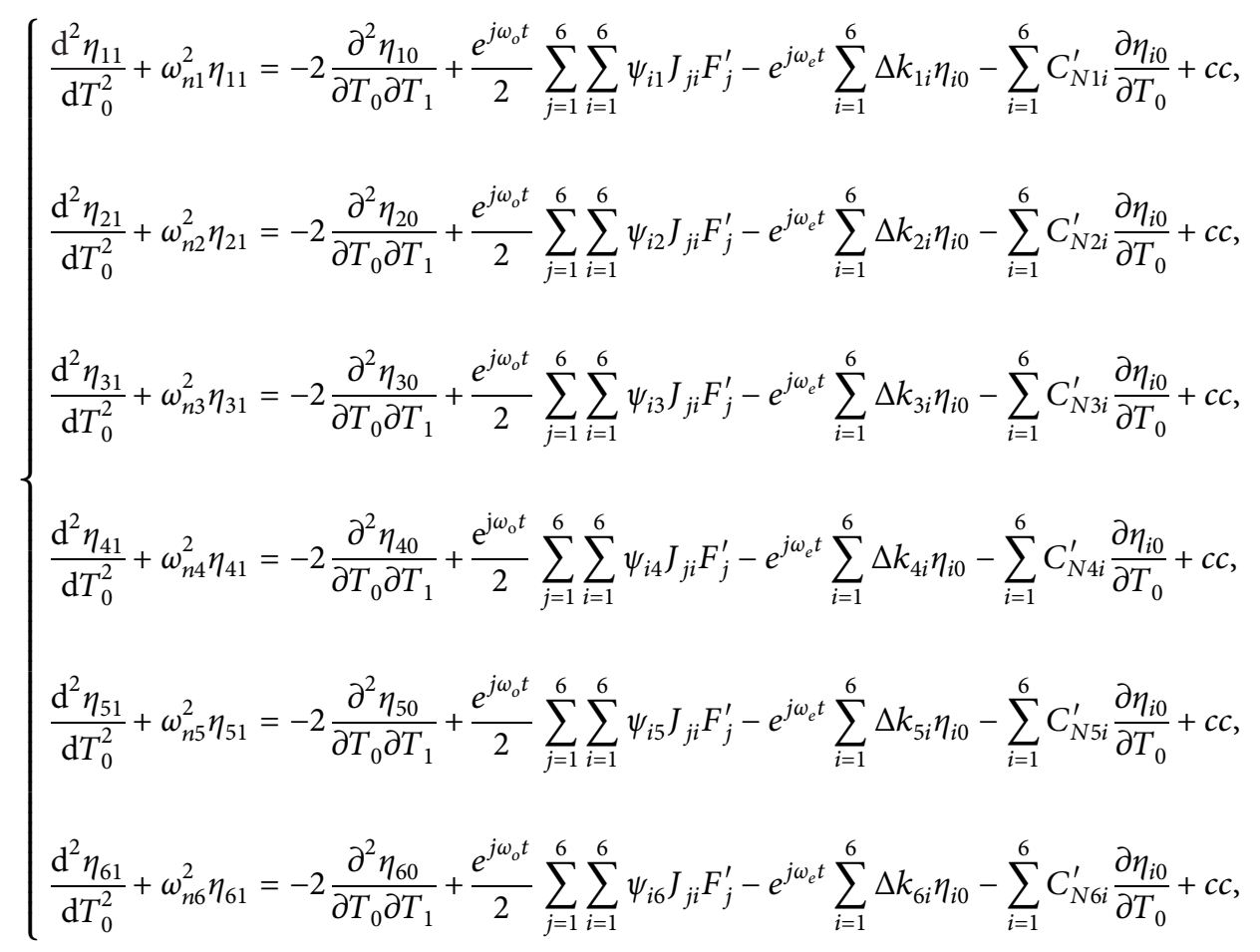

where $c c$ represents the complex conjugate.

Let the solution of equation (24) be

$$
\eta_{i 0}=A_{i} e^{j \omega_{n i} T_{0}}+c c
$$

where $A_{i}$ is an unknown function, and the solution can be substituted into equation (24):

$$
\left\{\begin{array}{l}
\frac{\mathrm{d}^{2} \eta_{11}}{\mathrm{~d} T_{0}^{2}}+\omega_{n 1}^{2} \eta_{11}=-2 j \omega_{n 1} \frac{d A_{1}}{d T_{1}} e^{j \omega_{n 1} T_{0}}+\frac{e^{j \omega_{0} t}}{2} \sum_{j=1}^{6} \sum_{i=1}^{6} \psi_{i 1} J_{j i} F_{j}^{\prime}-\sum_{i=1}^{6} C_{N 1 i}^{\prime} j \omega_{n i} A_{i} e^{j \omega_{n i} T_{0}}-\sum_{i=1}^{6} \Delta k_{1 i} A_{i} e^{j\left(\omega_{n i}+\omega_{e}\right) T_{0}}+c c \\
\frac{\mathrm{d}^{2} \eta_{21}}{\mathrm{~d} T_{0}^{2}}+\omega_{n 2}^{2} \eta_{21}=-2 j \omega_{n 2} \frac{d A_{1}}{d T_{1}} e^{j \omega_{n 2} T_{0}}+\frac{e^{j \omega_{o} t}}{2} \sum_{j=1}^{6} \sum_{i=1}^{6} \psi_{i 2} J_{j i} F_{j}^{\prime}-\sum_{i=1}^{6} C_{N 2 i}^{\prime} j \omega_{n i} A_{i} e^{j \omega_{n i} T_{0}}-\sum_{i=1}^{6} \Delta k_{2 i} A_{i} e^{j\left(\omega_{n i}+\omega_{e}\right) T_{0}}+c c \\
\frac{\mathrm{d}^{2} \eta_{31}}{\mathrm{~d} T_{0}^{2}}+\omega_{n 3}^{2} \eta_{31}=-2 j \omega_{n 3} \frac{d A_{1}}{d T_{1}} e^{j \omega_{n 3} T_{0}}+\frac{e^{j \omega_{0} t}}{2} \sum_{j=1}^{6} \sum_{i=1}^{6} \psi_{i 3} J_{j i} F_{j}^{\prime}-\sum_{i=1}^{6} C_{N 3 i}^{\prime} j \omega_{n i} A_{i} e^{j \omega_{n i} T_{0}}-\sum_{i=1}^{6} \Delta k_{3 i} A_{i} e^{j\left(\omega_{n i}+\omega_{e}\right) T_{0}}+c c \\
\frac{\mathrm{d}^{2} \eta_{41}}{\mathrm{~d} T_{0}^{2}}+\omega_{n 4}^{2} \eta_{41}=-2 j \omega_{n 4} \frac{d A_{1}}{d T_{1}} e^{j \omega_{n 4} T_{0}}+\frac{e^{j \omega_{0} t}}{2} \sum_{j=1}^{6} \sum_{i=1}^{6} \psi_{i 4} J_{j i} F_{j}^{\prime}-\sum_{i=1}^{6} C_{N 4 i}^{\prime} j \omega_{n i} A_{i} e^{j \omega_{n i} T_{0}}-\sum_{i=1}^{6} \Delta k_{4 i} A_{i} e^{j\left(\omega_{n i}+\omega_{e}\right) T_{0}}+c c \\
\frac{\mathrm{d}^{2} \eta_{51}}{\mathrm{~d} T_{0}^{2}}+\omega_{n 5}^{2} \eta_{51}=-2 j \omega_{n 5} \frac{d A_{1}}{d T_{1}} e^{j \omega_{n 5} T_{0}}+\frac{e^{j \omega_{0} t}}{2} \sum_{j=1}^{6} \sum_{i=1}^{6} \psi_{i 5} J_{j i} F_{j}^{\prime}-\sum_{i=1}^{6} C_{N 5 i}^{\prime} j \omega_{n i} A_{i} e^{j \omega_{n i} T_{0}}-\sum_{i=1}^{6} \Delta k_{5 i} A_{i} e^{j\left(\omega_{n i}+\omega_{e}\right) T_{0}}+c c \\
\frac{\mathrm{d}^{2} \eta_{61}}{\mathrm{~d} T_{0}^{2}}+\omega_{n 6}^{2} \eta_{61}=-2 j \omega_{n 6} \frac{d A_{1}}{d T_{1}} e^{j \omega_{n 6} T_{0}}+\frac{e^{j \omega_{0} t}}{2} \sum_{j=1}^{6} \sum_{i=1}^{6} \psi_{i 6} J_{j i} F_{j}^{\prime}-\sum_{i=1}^{6} C_{N 6 i}^{\prime} j \omega_{n i} A_{i} e^{j \omega_{n i} T_{0}}-\sum_{i=1}^{6} \Delta k_{6 i} A_{i} e^{j\left(\omega_{n i}+\omega_{e}\right) T_{0}}+c c
\end{array}\right.
$$


When $\omega_{\mathrm{o}}$ approaches $\omega_{n 1}$, the harmonic parameter $\sigma$ is introduced, and the frequency relationship of each angle is shown in the following formula:

$$
\omega_{o}=\omega_{n 1}+\varepsilon \sigma
$$

Substitute equation (27) into equation (26) and eliminate the perpetual term:

$$
\left\{\begin{array}{l}
-j \omega_{n 1}\left(2 \frac{\mathrm{d} A_{1}}{\mathrm{~d} T_{1}}+c_{N 11}^{\prime} A_{1}\right)+\sum_{j=1}^{6} \sum_{i=1}^{6} \psi_{i 1} J_{j i} F_{j}^{\prime} \frac{e^{j \sigma T_{1}}}{2}=0 \\
-j \omega_{n 2}\left(2 \frac{\mathrm{d} A_{2}}{\mathrm{~d} T_{1}}+c_{N 22}^{\prime} A_{2}\right)=0 \\
-j \omega_{n 3}\left(2 \frac{\mathrm{d} A_{3}}{\mathrm{~d} T_{1}}+c_{N 33}^{\prime} A_{3}\right)=0 \\
-j \omega_{n 4}\left(2 \frac{\mathrm{d} A_{4}}{\mathrm{~d} T_{1}}+c_{N 44}^{\prime} A_{4}\right)=0 \\
-j \omega_{n 5}\left(2 \frac{\mathrm{d} A_{5}}{\mathrm{~d} T_{1}}+c_{N 55}^{\prime} A_{5}\right)=0 \\
-j \omega_{n 6}\left(2 \frac{\mathrm{d} A_{6}}{\mathrm{~d} T_{1}}+c_{N 66}^{\prime} A_{6}\right)=0 .
\end{array}\right.
$$

The solution of equation (28) is obtained by using the constant conversion method:

$$
\left\{\begin{array}{l}
A_{1}=C_{1} e^{-\left(C_{N 11}^{\prime} T_{1} / 2\right)}+\frac{\sum_{j=1}^{6} \sum_{i=1}^{6} \psi_{i 1} J_{j i} F_{J}^{\prime}}{j 2 \omega_{n 1}\left(2 J \sigma+C_{N 11}^{\prime}\right.} e^{j \sigma T_{1}} \\
A_{2}=C_{2} e^{-\left(C_{N 22}^{\prime} T_{1} / 2\right)} \\
A_{3}=C_{3} e^{-\left(C_{N 33}^{\prime} T_{1} / 2\right)} \\
A_{4}=C_{4} e^{-\left(C_{N 44}^{\prime} T_{1} / 2\right)} \\
A_{5}=C_{5} e^{-\left(C_{N 55}^{\prime} T_{1} / 2\right)} \\
A_{6}=C_{6} e^{-\left(C_{N 66}^{\prime} T_{1} / 2\right)}
\end{array}\right.
$$

where $C_{1}, \ldots, C_{6}$ are constant.

By using the relationship between trigonometric functions and complex numbers and combining with Euler's formula, the first equation in equation (29) can be reduced to

$$
A_{1}=C_{1} e^{-\left(C_{N 11}^{\prime} / 2\right) T_{1}}-\frac{\sum_{j=1}^{6} \sum_{i=1}^{6} \psi_{i 1} J_{j i} F_{J}^{\prime}}{2 \omega_{n 1} \sqrt{\left(C_{N 11}^{\prime}\right)^{2}+4 \sigma^{2}}} e^{j\left(\theta+\sigma T_{1}\right)},
$$

where $\theta=\arctan \left(C_{N 11}^{\prime} / 2 \sigma\right)$.

With the increase of time, equation (29) will gradually approach zero. Therefore, by substituting equation (29) into equation (25), the steady-state zeroth degree approximate analytical solution of the electro-hydraulic platform system can be obtained as 


$$
\left\{\begin{array}{l}
\eta_{10}=-\frac{\sum_{j=1}^{6} \sum_{i=1}^{6} \psi_{i 1} J_{j i} F_{J}^{\prime}}{\omega_{n 1} \sqrt{\left(C_{N 11}^{\prime}\right)^{2}+4 \sigma^{2}} \cos \left(\theta+\left(\omega_{n 1}+\varepsilon \sigma\right) t\right)} \\
\eta_{20}=0 \\
\eta_{30}=0 \\
\eta_{40}=0 \\
\eta_{50}=0 \\
\eta_{60}=0 .
\end{array}\right.
$$

Substituting equations (29) and (30) into equation (26), the first-order approximate analytical solution of the steadystate electro-hydraulic system can be obtained as

$$
\begin{aligned}
& \eta_{11}=-2 \Delta k_{N 11} A_{1}\left(\frac{\cos \left(\omega_{n 1}-\omega_{e}\right) t}{\omega_{e}\left(2 \omega_{n 1}-\omega_{e}\right)}-\frac{\cos \left(\omega_{n 1}-\omega_{e}\right) t}{\omega_{e}\left(2 \omega_{n 1}+\omega_{e}\right)}\right) \text {, } \\
& \eta_{21}=\frac{\sum_{j=1}^{6} \sum_{i=1}^{6} \psi_{i 2} J_{j i} F_{J}^{\prime} \cos \left(\omega_{0} t\right)}{\omega_{n 2}^{2}-\omega_{0}^{2}}-2\left[\begin{array}{c}
\Delta k_{N 21} A_{1}\left(\frac{\cos \left(\omega_{n 1}+\omega_{e}\right) t}{\omega_{n 2}^{2}-\left(\omega_{n 1}+\omega_{e}\right)^{2}}+\frac{\cos \left(\omega_{n 1}-\omega_{e}\right) t}{\omega_{n 2}^{2}-\left(\omega_{n 1}-\omega_{e}\right)^{2}}\right) \\
-\frac{C_{N 21}^{\prime} \omega_{n 1} A_{1} \sin \omega_{n 1} t}{\omega_{n 2}^{2}-\omega_{n 1}^{2}}
\end{array}\right], \\
& \eta_{31}=\frac{\sum_{j=1}^{6} \sum_{i=1}^{6} \psi_{i 3} J_{j i} F_{J}^{\prime} \cos \left(\omega_{0} t\right)}{\omega_{n 3}^{2}-\omega_{0}^{2}}-2\left[\begin{array}{c}
\Delta k_{N 31} A_{1}\left(\frac{\cos \left(\omega_{n 1}+\omega_{e}\right) t}{\omega_{n 3}^{2}-\left(\omega_{n 1}+\omega_{e}\right)^{2}}+\frac{\cos \left(\omega_{n 1}-\omega_{e}\right) t}{\omega_{n 3}^{2}-\left(\omega_{n 1}-\omega_{e}\right)^{2}}\right) \\
-\frac{C_{N 31}^{\prime} \omega_{n 1} A_{1} \sin \omega_{n 1} t}{\omega_{n 3}^{2}-\omega_{n 1}^{2}}
\end{array}\right], \\
& \eta_{41}=\frac{\sum_{j=1}^{6} \sum_{i=1}^{6} \psi_{i 4} J_{j i} F_{J}^{\prime} \cos \left(\omega_{0} t\right)}{\omega_{n 4}^{2}-\omega_{0}^{2}}-2\left[\begin{array}{c}
\Delta k_{N 41} A_{1}\left(\frac{\cos \left(\omega_{n 1}+\omega_{e}\right) t}{\omega_{n 4}^{2}-\left(\omega_{n 1}+\omega_{e}\right)^{2}}+\frac{\cos \left(\omega_{n 1}-\omega_{e}\right) t}{\omega_{n 4}^{2}-\left(\omega_{n 1}-\omega_{e}\right)^{2}}\right) \\
-\frac{C_{N 41}^{\prime} \omega_{n 1} A_{1} \sin \omega_{n 1} t}{\omega_{n 4}^{2}-\omega_{n 1}^{2}}
\end{array}\right], \\
& \eta_{51}=\frac{\sum_{j=1}^{6} \sum_{i=1}^{6} \psi_{i 5} J_{j i} F_{J}^{\prime} \cos \left(\omega_{0} t\right)}{\omega_{n 5}^{2}-\omega_{0}^{2}}-2\left[\begin{array}{c}
\Delta k_{N 51} A_{1}\left(\frac{\cos \left(\omega_{n 1}+\omega_{e}\right) t}{\omega_{n 5}^{2}-\left(\omega_{n 1}+\omega_{e}\right)^{2}}+\frac{\cos \left(\omega_{n 1}-\omega_{e}\right) t}{\omega_{n 5}^{2}-\left(\omega_{n 1}-\omega_{e}\right)^{2}}\right) \\
-\frac{C_{N 51}^{\prime} \omega_{n 1} A_{1} \sin \omega_{n 1} t}{\omega_{n 5}^{2}-\omega_{n 1}^{2}}
\end{array}\right], \\
& \eta_{61}=\frac{\sum_{j=1}^{6} \sum_{i=1}^{6} \psi_{i 6} J_{j i} F_{J}^{\prime} \cos \left(\omega_{0} t\right)}{\omega_{n 6}^{2}-\omega_{0}^{2}}-2\left[\begin{array}{c}
\Delta k_{N 61} A_{1}\left(\frac{\cos \left(\omega_{n 1}+\omega_{e}\right) t}{\omega_{n 6}^{2}-\left(\omega_{n 1}+\omega_{e}\right)^{2}}+\frac{\cos \left(\omega_{n 1}-\omega_{e}\right) t}{\omega_{n 6}^{2}-\left(\omega_{n 1}-\omega_{e}\right)^{2}}\right) \\
-\frac{C_{N 61}^{\prime} \omega_{n 1} A_{1} \sin \omega_{n 1} t}{\omega_{n 6}^{2}-\omega_{n 1}^{2}}
\end{array}\right] .
\end{aligned}
$$


Then, the steady-state response of the main resonance of the electro-hydraulic platform system in rectangular coordinates is

$$
x=\psi\left(\eta_{0}+\varepsilon \eta_{1}\right) .
$$

In the same way, the main resonance responses can be obtained, while the external excitation frequency approaches to the second-order to the sixth-order natural frequency of the current electro-hydraulic platform system.

4.2. Approximate Analytical Derivation of Combined Resonance of Electro-Hydraulic Platform Transmission System.
The combined resonance response of the electro-hydraulic platform transmission system is derived by the multiscale method, which can be expressed as

$$
\eta_{i}\left(T_{0}, T_{1}\right)=\eta_{i 0}\left(T_{0}, T_{1}\right)+\varepsilon \eta_{i 1}\left(T_{0}, T_{1}\right),
$$

where $T_{0}=t$ and $T_{1}=\varepsilon t$.

A small parameter is introduced into the damping:

$$
c_{N i j}=\varepsilon c_{N i j}^{\prime} \text {. }
$$

Substituting equations (34) and (35) into equation (20), the zeroth power term is sorted out and the equation is shown as follows:

$$
\left\{\begin{array}{l}
\frac{\partial^{2} \eta_{10}}{\partial T_{0}^{2}}+\omega_{n 1}^{2} \eta_{10}=\frac{e^{j \omega_{o} T_{0}}}{2} \sum_{j=1}^{6} \sum_{i=1}^{6} \psi_{i 1} J_{j i} F_{j}+c c \\
\frac{\partial^{2} \eta_{20}}{\partial T_{0}^{2}}+\omega_{n 2}^{2} \eta_{20}=\frac{e^{j \omega_{o} T_{0}}}{2} \sum_{j=1}^{6} \sum_{i=1}^{6} \psi_{i 2} J_{j i} F_{j}+c c \\
\frac{\partial^{2} \eta_{30}}{\partial T_{0}^{2}}+\omega_{n 3}^{2} \eta_{30}=\frac{e^{j \omega_{o} T_{0}}}{2} \sum_{j=1}^{6} \sum_{i=1}^{6} \psi_{i 3} J_{j i} F_{j}+c c \\
\frac{\partial^{2} \eta_{40}}{\partial T_{0}^{2}}+\omega_{n 4}^{2} \eta_{40}=\frac{e^{j \omega_{o} T_{0}}}{2} \sum_{j=1}^{6} \sum_{i=1}^{6} \psi_{i 4} J_{j i} F_{j}+c c \\
\frac{\partial^{2} \eta_{50}}{\partial T_{0}^{2}}+\omega_{n 5}^{2} \eta_{50}=\frac{e^{j \omega_{o} T_{0}}}{2} \sum_{j=1}^{6} \sum_{i=1}^{6} \psi_{i 5} J_{j i} F_{j}+c c \\
\frac{\partial^{2} \eta_{60}}{\partial T_{0}^{2}}+\omega_{n 6}^{2} \eta_{60}=\frac{e^{j \omega_{o} T_{0}}}{2} \sum_{j=1}^{6} \sum_{i=1}^{6} \psi_{i 6} J_{j i} F_{j}+c c .
\end{array}\right.
$$


The equation for sorting out the first power term is shown as follows:

$$
\left\{\begin{array}{l}
\frac{\partial^{2} \eta_{11}}{\partial T_{0}^{2}}+\omega_{n 1}^{2} \eta_{11}=-2 \frac{\partial^{2} \eta_{10}}{\partial T_{0} \partial T_{1}}-e^{j \omega_{e} t} \sum_{i=1}^{6} \Delta k_{1 i} \eta_{i 0}-\sum_{i=1}^{6} C_{N 1 i}^{\prime} \frac{\partial \eta_{i 0}}{\partial T_{0}}+c c \\
\frac{\partial^{2} \eta_{21}}{\partial T_{0}^{2}}+\omega_{n 2}^{2} \eta_{21}=-2 \frac{\partial^{2} \eta_{20}}{\partial T_{0} \partial T_{1}}-e^{j \omega_{e} t} \sum_{i=1}^{6} \Delta k_{2 i} \eta_{i 0}-\sum_{i=1}^{6} C_{N 2 i}^{\prime} \frac{\partial \eta_{i 0}}{\partial T_{0}}+c c \\
\frac{\partial^{2} \eta_{31}}{\partial T_{0}^{2}}+\omega_{n 3}^{2} \eta_{31}=-2 \frac{\partial^{2} \eta_{30}}{\partial T_{0} \partial T_{1}}-e^{j \omega_{e} t} \sum_{i=1}^{6} \Delta k_{3 i} \eta_{i 0}-\sum_{i=1}^{6} C_{N 3 i}^{\prime} \frac{\partial \eta_{i 0}}{\partial T_{0}}+c c \\
\frac{\partial^{2} \eta_{41}}{\partial T_{0}^{2}}+\omega_{n 4}^{2} \eta_{41}=-2 \frac{\partial^{2} \eta_{40}}{\partial T_{0} \partial T_{1}}-e^{j \omega_{e} t} \sum_{i=1}^{6} \Delta k_{4 i} \eta_{i 0}-\sum_{i=1}^{6} C_{N 4 i}^{\prime} \frac{\partial \eta_{i 0}}{\partial T_{0}}+c c \\
\frac{\partial^{2} \eta_{51}}{\partial T_{0}^{2}}+\omega_{n 5}^{2} \eta_{51}=-2 \frac{\partial^{2} \eta_{50}}{\partial T_{0} \partial T_{1}}-e^{j \omega_{e} t} \sum_{i=1}^{6} \Delta k_{5 i} \eta_{i 0}-\sum_{i=1}^{6} C_{N 5 i}^{\prime} \frac{\partial \eta_{i 0}}{\partial T_{0}}+c c \\
\frac{\partial^{2} \eta_{61}}{\partial T_{0}^{2}}+\omega_{n 6}^{2} \eta_{61}=-2 \frac{\partial^{2} \eta_{60}}{\partial T_{0} \partial T_{1}}-e^{j \omega_{e} t} \sum_{i=1}^{6} \Delta k_{6 i} \eta_{i 0}-\sum_{i=1}^{6} C_{N 6 i}^{\prime} \frac{\partial \eta_{i 0}}{\partial T_{0}}+c c
\end{array}\right.
$$

where $c c r e p r e s e n t s$ the complex conjugate.

Let the analytic solution of equation (37) be as follows:

$$
\eta_{i 0}=B_{i} e^{j \omega_{n i} T_{0}}+D_{i} e^{j \omega_{0} T_{0}}+c c
$$

where $D_{i}=\left(\left(\sum_{j=1}^{6} \sum_{i=1}^{6} \psi_{i 1} J_{j i} F_{j}\right) / 2\left(\omega_{\mathrm{n} i}^{2}-\omega_{\mathrm{o}}^{2}\right)\right)$.

Substitute equation (38) into equation (37) to obtain the equation as shown below:

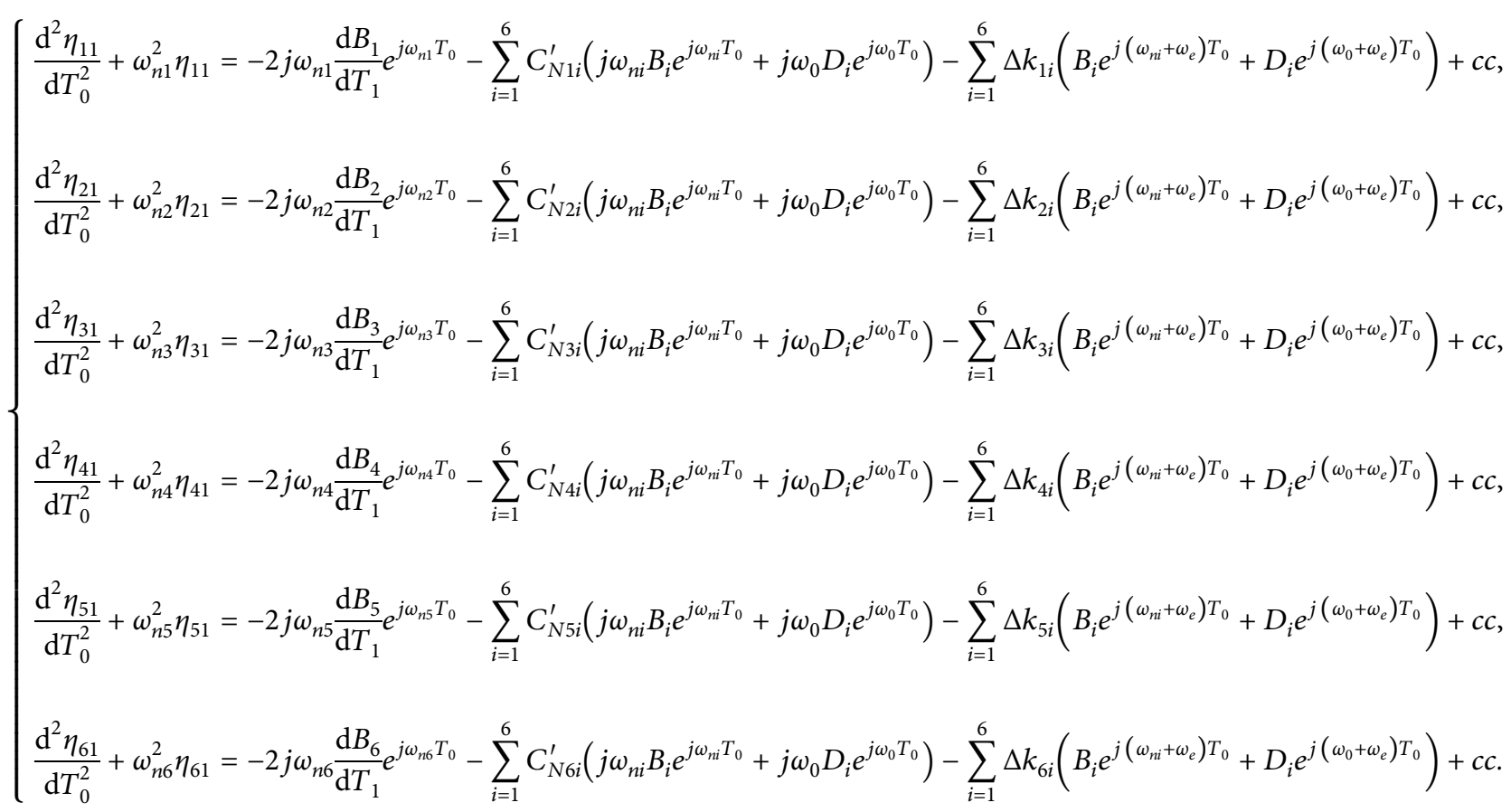


By analyzing the above equation, we can see that when $\omega_{o} \approx \omega_{n i}$, the main resonance occurs in the system. In addition, when $\omega_{0} \approx \omega_{n i} \pm \omega_{e}$, the system will also have combinatorial resonance. Under the condition that the external excitation frequency approaches to the sum of the first-order natural frequency and the equivalent stiffness pulsation frequency of hydraulic oil, the harmonic parameters are introduced as follows:

$$
\omega_{0}=\omega_{n 1}+\omega_{e}+\varepsilon \sigma
$$

Substitute equation (40) into equation (39) and eliminate the perpetual term:

$$
\left\{\begin{array}{l}
2 j \omega_{n 1}\left(2 \frac{d B_{1}}{d T_{1}}+C_{N 11}^{\prime} B_{1}\right)+\sum_{i=1}^{6} \Delta k_{1 i} D_{i} e^{j \sigma T_{1}}=0 \\
2 j \omega_{n 2}\left(2 \frac{\mathrm{d} B_{2}}{\mathrm{~d} T_{1}}+C_{N 22}^{\prime} B_{2}\right)=0 \\
2 j \omega_{n 3}\left(2 \frac{\mathrm{d} B_{3}}{\mathrm{~d} T_{1}}+C_{N 33}^{\prime} B_{3}\right)=0 \\
2 j \omega_{n 4}\left(2 \frac{\mathrm{d} B_{4}}{\mathrm{~d} T_{1}}+C_{N 44}^{\prime} B_{4}\right)=0 \\
2 j \omega_{n 5}\left(2 \frac{\mathrm{d} B_{5}}{\mathrm{~d} T_{1}}+C_{N 55}^{\prime} B_{5}\right)=0 \\
2 j \omega_{n 6}\left(2 \frac{\mathrm{d} B_{6}}{\mathrm{~d} T_{1}}+C_{N 66}^{\prime} B_{6}\right)=0 .
\end{array}\right.
$$

The general solution of equation (41) is

$$
\left\{\begin{array}{l}
B_{1}=E_{1} e^{-\left(C_{N 11}^{\prime} / 2\right) T_{1}}+\frac{\sum_{i=1}^{6} \Delta k_{N 1 i} D_{i}}{\omega_{n 1} \sqrt{4 \sigma^{2}+\left(C_{N 11}^{\prime}\right)^{2}}} e^{j\left(\theta+\sigma T_{1}\right)}, \\
B_{2}=E_{2} e^{-\left(C_{N 22}^{\prime} / 2\right) T_{1}} \\
B_{3}=E_{3} e^{-\left(C_{N 3}^{\prime} / 2\right) T_{1}}, \\
B_{4}=E_{4} e^{-\left(C_{N 44}^{\prime} / 2\right) T_{1}} \\
B_{5}=E_{5} e^{-\left(C_{N 55}^{\prime} / 2\right) T_{1}} \\
B_{6}=E_{6} e^{-\left(C_{N 66}^{\prime} / 2\right) T_{1}}
\end{array}\right.
$$

where $E_{1}, \ldots, E_{6}$ are constant and $\theta=\arctan \left(C_{N 11}^{\prime} / 2 \sigma\right)$.

Similar to the principal vibration analysis, by substituting equation (42) into equation (38), the zeroth degree approximate analytical solution of the combined resonance of the 6-DOF platform system can be obtained as follows:

$$
\left\{\begin{array}{l}
\eta_{10}=2 \times \frac{\sum_{i=1}^{6} \Delta k_{N 1 i} D_{i}}{\omega_{n 1} \sqrt{4 \sigma^{2}+\left(C_{N 11}^{\prime}\right)^{2}}} \cos \left[\theta+\left(\omega_{n 1}+\varepsilon \sigma\right) t\right]+2 D_{1} \cos \omega_{0} t \\
\eta_{20}=2 D_{2} \cos \omega_{0} t \\
\eta_{30}=2 D_{3} \cos \omega_{0} t \\
\eta_{40}=2 D_{4} \cos \omega_{0} t \\
\eta_{50}=2 D_{5} \cos \omega_{0} t \\
\eta_{60}=2 D_{6} \cos \omega_{0} t .
\end{array}\right.
$$

Substituting equation (42) into equation (39), the steadystate first-order approximate analytical solution of the combined resonance of the transmission system can be obtained as follows:

$$
\left\{\begin{array}{l}
\eta_{11}=-2\left(\eta_{a 1}+\eta_{b 1}-\eta_{c 1}\right), \\
\eta_{21}=-2\left[\eta_{a 2}+\eta_{b 2}-\eta_{c 2}-\frac{\left(\omega_{n 1} C_{N 21}^{\prime} B_{1} \sin \omega_{n 1} t\right)}{\omega_{n 2}^{2}-\omega_{n 1}^{2}}\right] \\
\eta_{31}=-2\left[\eta_{a 3}+\eta_{b 3}-\eta_{c 3}-\frac{\left(\omega_{n 1} C_{N 31}^{\prime} B_{1} \sin \omega_{n 1} t\right)}{\omega_{n 3}^{2}-\omega_{n 1}^{2}}\right], \\
\eta_{21}=-2\left[\eta_{a 4}+\eta_{b 4}-\eta_{c 4}-\frac{\left(\omega_{n 1} C_{N 41}^{\prime} B_{1} \sin \omega_{n 1} t\right)}{\omega_{n 4}^{2}-\omega_{n 1}^{2}}\right], \\
\eta_{21}=-2\left[\eta_{a 5}+\eta_{b 5}-\eta_{c 5}-\frac{\left(\omega_{n 1} C_{N 51}^{\prime} B_{1} \sin \omega_{n 1} t\right)}{\omega_{n 5}^{2}-\omega_{n 1}^{2}}\right], \\
\eta_{21}=-2\left[\eta_{a 6}+\eta_{b 6}-\eta_{c 6}-\frac{\left(\omega_{n 1} C_{N 61}^{\prime} B_{1} \sin \omega_{n 1} t\right)}{\omega_{n 6}^{2}-\omega_{n 1}^{2}}\right],
\end{array}\right.
$$

where 


$$
\begin{aligned}
& \eta_{a 1}=\Delta k_{N 11} B_{1}\left[\frac{\cos \left(\omega_{n 1}-\omega_{f}\right) t}{\omega_{f}\left(2 \omega_{n 1}-\omega_{f}\right)}-\frac{\cos \left(\omega_{n 1}+\omega_{f}\right) t}{\omega_{f}\left(2 \omega_{n 1}+\omega_{f}\right)}\right], \\
& \eta_{a 2}=\Delta k_{N 21} B_{1}\left[\frac{\cos \left(\omega_{n 1}+\omega_{f}\right) t}{\omega_{n 2}^{2}-\left(\omega_{n 1}+\omega_{f}\right)^{2}}-\frac{\cos \left(\omega_{n 1}-\omega_{f}\right) t}{\omega_{n 2}^{2}-\left(\omega_{n 1}-\omega_{f}\right)^{2}}\right] \text {, } \\
& \eta_{a 3}=\Delta k_{N 31} B_{1}\left[\frac{\cos \left(\omega_{n 1}+\omega_{f}\right) t}{\omega_{n 3}^{2}-\left(\omega_{n 1}+\omega_{f}\right)^{2}}-\frac{\cos \left(\omega_{n 1}-\omega_{f}\right) t}{\omega_{n 3}^{2}-\left(\omega_{n 1}-\omega_{f}\right)^{2}}\right] \text {, } \\
& \eta_{a 4}=\Delta k_{N 41} B_{1}\left[\frac{\cos \left(\omega_{n 1}+\omega_{f}\right) t}{\omega_{n 4}^{2}-\left(\omega_{n 1}+\omega_{f}\right)^{2}}-\frac{\cos \left(\omega_{n 1}-\omega_{f}\right) t}{\omega_{n 4}^{2}-\left(\omega_{n 1}-\omega_{f}\right)^{2}}\right] \text {, } \\
& \eta_{a 5}=\Delta k_{N 51} B_{1}\left[\frac{\cos \left(\omega_{n 1}+\omega_{f}\right) t}{\omega_{n 5}^{2}-\left(\omega_{n 1}+\omega_{f}\right)^{2}}-\frac{\cos \left(\omega_{n 1}-\omega_{f}\right) t}{\omega_{n 5}^{2}-\left(\omega_{n 1}-\omega_{f}\right)^{2}}\right] \text {, } \\
& \eta_{a 6}=\Delta k_{N 61} B_{1}\left[\frac{\cos \left(\omega_{n 1}+\omega_{f}\right) t}{\omega_{n 6}^{2}-\left(\omega_{n 1}+\omega_{f}\right)^{2}}-\frac{\cos \left(\omega_{n 1}-\omega_{f}\right) t}{\omega_{n 6}^{2}-\left(\omega_{n 1}-\omega_{f}\right)^{2}}\right] \text {, } \\
& \eta_{b 1}=\frac{\sum_{i=1}^{6} \Delta k_{N 1 i} D_{i} \cos \left(\omega_{n 1}-\omega_{f}\right) t}{\omega_{n 1}^{2}-\left(\omega_{n 1}+\omega_{f}\right)^{2}}, \\
& \eta_{b 2}=\sum_{i=1}^{6} \Delta k_{N 2 i} D_{i}\left[\frac{\cos \left(\omega_{n 1}+\omega_{f}\right) t}{\omega_{n 2}^{2}-\left(\omega_{n 1}+\omega_{f}\right)^{2}}+\frac{\cos \left(\omega_{n 1}-\omega_{f}\right) t}{\omega_{n 2}^{2}-\left(\omega_{n 1}-\omega_{f}\right)^{2}}\right] \text {, } \\
& \eta_{b 3}=\sum_{i=1}^{6} \Delta k_{N 3 i} D_{i}\left[\frac{\cos \left(\omega_{n 1}+\omega_{f}\right) t}{\omega_{n 3}^{2}-\left(\omega_{n 1}+\omega_{f}\right)^{2}}+\frac{\cos \left(\omega_{n 1}-\omega_{f}\right) t}{\omega_{n 3}^{2}-\left(\omega_{n 1}-\omega_{f}\right)^{2}}\right] \text {, } \\
& \eta_{b 4}=\sum_{i=1}^{6} \Delta k_{N 4 i} D_{i}\left[\frac{\cos \left(\omega_{n 1}+\omega_{f}\right) t}{\omega_{n 4}^{2}-\left(\omega_{n 1}+\omega_{f}\right)^{2}}+\frac{\cos \left(\omega_{n 1}-\omega_{f}\right) t}{\omega_{n 4}^{2}-\left(\omega_{n 1}-\omega_{f}\right)^{2}}\right] \text {, } \\
& \eta_{b 5}=\sum_{i=1}^{6} \Delta k_{N 5 i} D_{i}\left[\frac{\cos \left(\omega_{n 1}+\omega_{f}\right) t}{\omega_{n 5}^{2}-\left(\omega_{n 1}+\omega_{f}\right)^{2}}+\frac{\cos \left(\omega_{n 1}-\omega_{f}\right) t}{\omega_{n 5}^{2}-\left(\omega_{n 1}-\omega_{f}\right)^{2}}\right] \text {, } \\
& \eta_{b 6}=\sum_{i=1}^{6} \Delta k_{N 6 i} D_{i}\left[\frac{\cos \left(\omega_{n 1}+\omega_{f}\right) t}{\omega_{n 6}^{2}-\left(\omega_{n 1}+\omega_{f}\right)^{2}}+\frac{\cos \left(\omega_{n 1}-\omega_{f}\right) t}{\omega_{n 6}^{2}-\left(\omega_{n 1}-\omega_{f}\right)^{2}}\right] \text {, } \\
& \eta_{c 1}=\frac{\omega_{0} \sum_{i=1}^{6} C_{N 1 i}^{\prime} D_{i} \sin \omega_{0} t}{\omega_{n 1}^{2}-\omega_{0}^{2}}, \\
& \eta_{c 2}=\frac{\omega_{0} \sum_{i=1}^{6} C_{N 2 i}^{\prime} D_{i} \sin \omega_{0} t}{\omega_{n 2}^{2}-\omega_{0}^{2}}, \\
& \eta_{c 3}=\frac{\omega_{0} \sum_{i=1}^{6} C_{N 3 i}^{\prime} D_{i} \sin \omega_{0} t}{\omega_{n 3}^{2}-\omega_{0}^{2}}, \\
& \eta_{c 4}=\frac{\omega_{0} \sum_{i=1}^{6} C_{N 4 i}^{\prime} D_{i} \sin \omega_{0} t}{\omega_{n 4}^{2}-\omega_{0}^{2}},
\end{aligned}
$$




$$
\begin{aligned}
& \eta_{c 5}=\frac{\omega_{0} \sum_{i=1}^{6} C_{N 5 i}^{\prime} D_{i} \sin \omega_{0} t}{\omega_{n 5}^{2}-\omega_{0}^{2}}, \\
& \eta_{c 6}=\frac{\omega_{0} \sum_{i=1}^{6} C_{N 6 i}^{\prime} D_{i} \sin \omega_{0} t}{\omega_{n 6}^{2}-\omega_{0}^{2}} .
\end{aligned}
$$

Then, the combined resonant steady-state response of the 6-DOF platform system in rectangular coordinates is

$$
x=\psi\left(\eta_{0}+\varepsilon \eta_{1}\right) .
$$

Similarly, the combined resonance response can be obtained by referring to the above solution process, while the external excitation frequency is close to the sum of the second order to the sixth-order natural frequency of the 6DOF platform system and the equivalent stiffness fluctuation frequency of the hydraulic oil unit.

\section{Parametric Vibration Response Analysis of an Electro-Hydraulic Stewart Platform}

5.1. Vibration Response Analysis of System Parameters in Pose 1. Position and pose 1 parameter:

$$
\begin{aligned}
\mathbf{x} & =\left(P_{x}, P_{y}, P_{z}, \alpha, \beta, \gamma\right)^{T} \\
& =\left(\begin{array}{llllll}
0 & 0 & 2 \sqrt{3} & 0 & 0 & 0
\end{array}\right)^{T} .
\end{aligned}
$$

When the platform is in the position of pose 1 , the vibration response of the system parameter is analyzed.

Parameter vibration-related calculation parameters of the 6-DOF platform transmission system are shown in Table 2. Substituting the platform mass, leg stiffness, and parameters shown in Table 1 into equation (20), the parametric vibration equation of the 6-DOF platform transmission system is obtained.

5.1.1. Main Resonance Response Analysis. The time-domain and frequency-domain responses of the main resonance when the external excitation frequency approaches to the stable value of the first-order to the sixth-order natural angular frequency of the electro-hydraulic platform transmission system are shown in Figures 2-7. As can be seen from Figures 2(a)-7(a), while the external excitation frequency approaches to the steady-state value of the natural frequency of the first to sixth order of the transmission system, respectively, the mode corresponding to the natural frequency of the system will have a relatively intense main resonance phenomenon, and its maximum displacement is $0.00002712 \mathrm{~mm}$. As can be seen from Figures 2(b)-7(b), when the main resonance is generated in the system, there is a spike in the frequencydomain image, which corresponds to the external excitation frequency close to the natural frequency. This indicates that it plays a leading role in the main resonance of the system. In this case, the dominant frequency is the sum of the natural frequencies of each order and the fluctuation frequency of the equivalent stiffness of hydraulic oil, and other natural frequencies also reach their maximum amplitudes at the dominant frequency.

5.1.2. Combined Resonance Response. While the external excitation frequency approaches to the combined frequency of the stable value of the first- to sixth-order natural angular frequency of the electro-hydraulic platform transmission system and the fluctuation angular frequency of the equivalent stiffness of hydraulic oil, the combined resonance time-domain response and frequency-domain response are shown in Figures 8-13(a). As can be seen from Figures 8(a)-13(a), the combined resonance phenomenon occurs in the system, but its amplitude is larger than that of the system when the main resonance occurs, and the maximum displacement is $0.09427 \mathrm{~mm}$. According to Figures 8(b)-13(b), when the system produces combined resonance, there are three spikes in the frequency-domain image. Among them, the combined frequency $\omega_{o} \approx \omega_{\mathrm{i}}+\omega_{e}$, which is the stable value of the natural angular frequency of each order of the system and the fluctuation angular frequency of the equivalent stiffness of hydraulic oil, has the highest peak, indicating that it plays a leading role in the combined resonance of the system, while the other frequencies play a regulating role. There is a lower peak on the left and right side of the peak corresponding to the combined frequencies, with the left corresponding frequency $\omega_{o} \approx \omega_{i}$ and the right corresponding frequency $\omega_{o} \approx \omega_{i}+2 \omega_{e}(i=1$, $2, \ldots, 6)$.

5.2. Vibration Response Analysis of System Parameters in Pose 2. Position and pose 2 parameter:

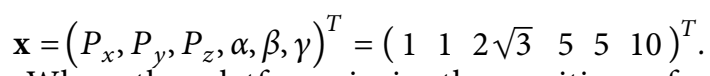

When the platform is in the position of pose 2 , the parameter vibration response of the system is analyzed.

Parameter vibration-related calculation parameters of the 6-DOF platform transmission system are shown in Table 3. Substituting the platform mass, leg stiffness, and parameters shown in Table 1 into equation (20), the parametric vibration equation of the 6-DOF platform transmission system is obtained.

5.2.1. Main Resonance Response Analysis. The time-domain and frequency-domain responses of the main resonance, while the external excitation frequency is close to the 
TABLE 2: Calculation parameters related to vibration of 6-DOF platform transmission system parameters.

\begin{tabular}{|c|c|}
\hline Name & Value \\
\hline Steady-state value of the first-order natural frequency $\omega_{1}\left(\mathrm{rad} \cdot \mathrm{s}^{-1}\right)$ & 128.4811 \\
\hline Steady-state value of the second-order natural frequency $\omega_{2}\left(\mathrm{rad} \cdot \mathrm{s}^{-1}\right)$ & 128.4811 \\
\hline Steady-state value of the third-order natural frequency $\omega_{3}\left(\mathrm{rad} \cdot \mathrm{s}^{-1}\right)$ & 259.8076 \\
\hline Steady-state value of the fourth-order natural frequency $\omega_{4}\left(\mathrm{rad} \cdot \mathrm{s}^{-1}\right)$ & 367.4235 \\
\hline Steady-state value of the fifth-order natural frequency $\omega_{5}\left(\mathrm{rad} \cdot \mathrm{s}^{-1}\right)$ & 524.4956 \\
\hline Steady-state value of the sixth-order natural frequency $\omega_{6}\left(\mathrm{rad} \cdot \mathrm{s}^{-1}\right)$ & 524.4956 \\
\hline Fluctuation frequency of equivalent stiffness of fluid element $\omega_{e}\left(\mathrm{rad} \cdot \mathrm{s}^{-1}\right)$ & 40.7150 \\
\hline Amplitude of external excitation $F_{0}(\mathrm{~N})$ & {$\left[\begin{array}{llllll}20 & 20 & 20 & 20 & 20 & 20\end{array}\right]^{T}$} \\
\hline Structural damping $C / N \cdot(\mathrm{m} / \mathrm{s})$ & {$\left[\begin{array}{llllll}20 & 20 & 20 & 20 & 20 & 20\end{array}\right]^{T}$} \\
\hline
\end{tabular}

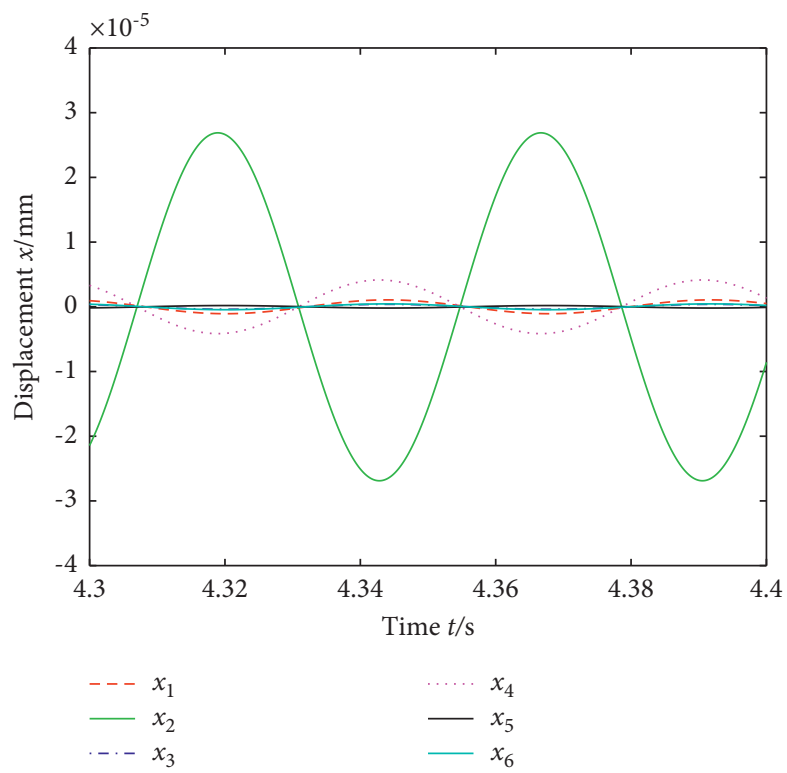

(a)
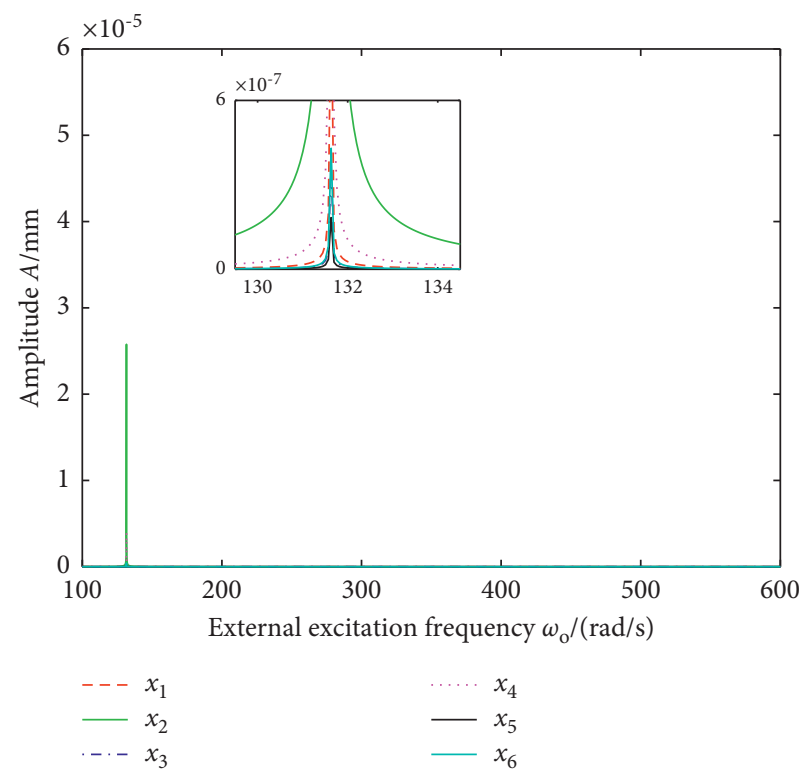

(b)

Figure 2: Main vibration response of the electro-hydraulic Stewart platform transmission system at $\omega_{o} \approx \omega_{1}$. (a) $\omega_{o} \approx \omega_{1}$ time-domain response. (b) $\omega_{o} \approx \omega_{1}$ frequency-domain response.

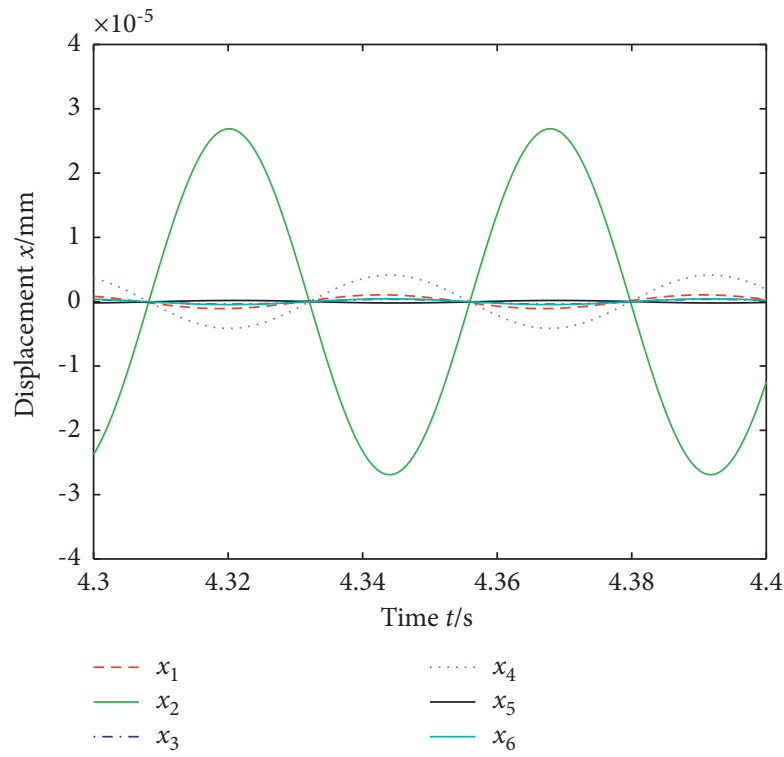

(a)

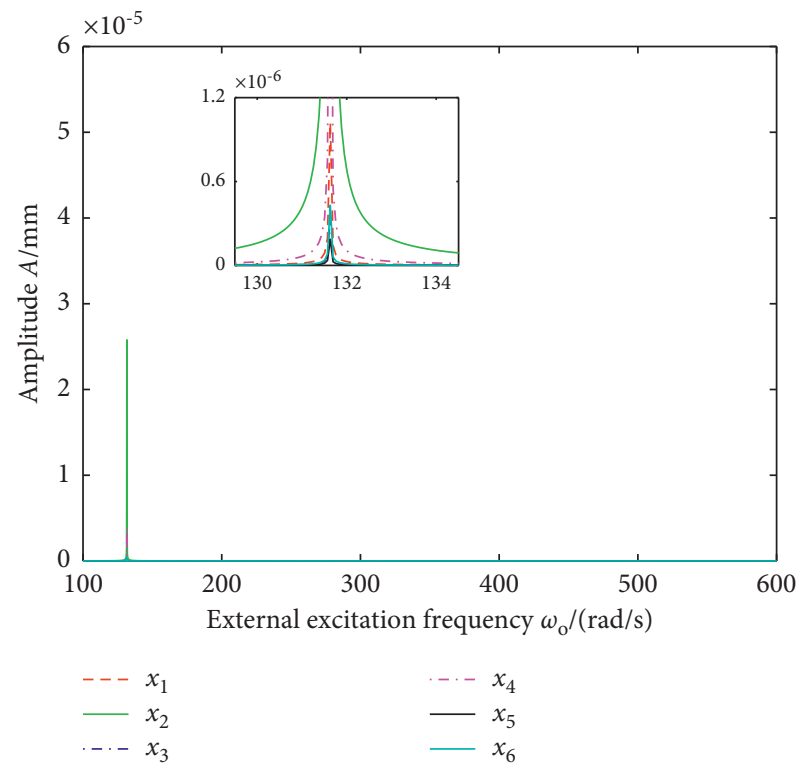

(b)

Figure 3: Main vibration response of the electro-hydraulic Stewart platform transmission system at $\omega_{o} \approx \omega_{2}$. (a) $\omega_{o} \approx \omega_{2}$ time-domain response. (b) $\omega_{o} \approx \omega_{2}$ frequency-domain response. 


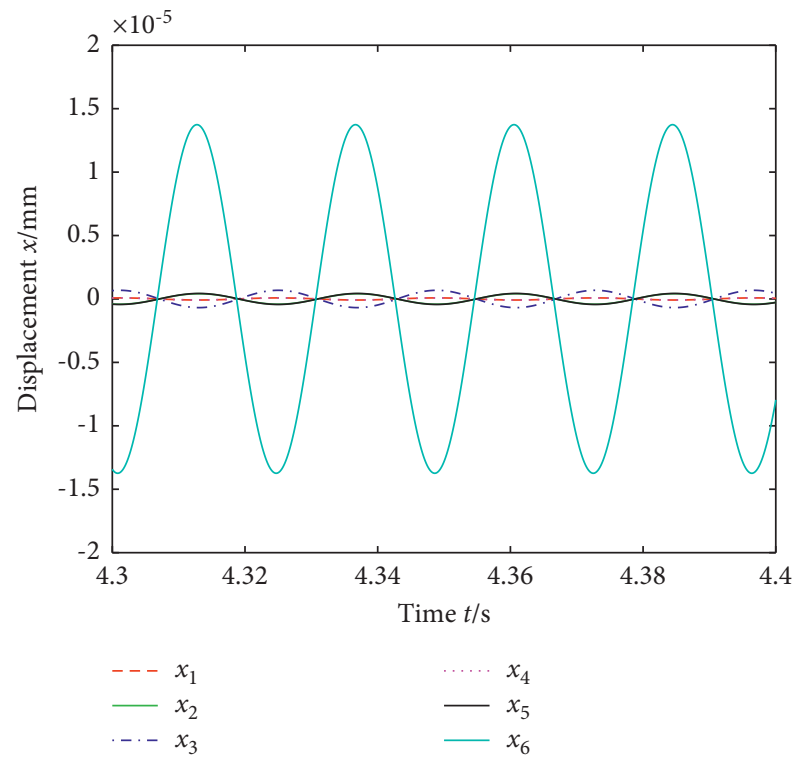

(a)

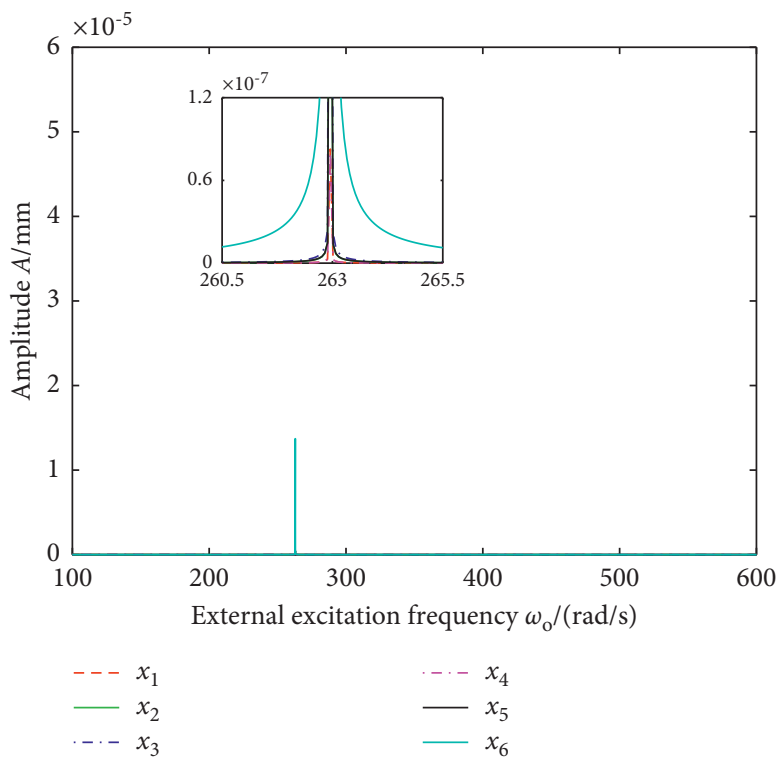

(b)

Figure 4: Main vibration response of the electro-hydraulic Stewart platform transmission system at $\omega_{o} \approx \omega_{3}$. (a) $\omega_{o} \approx \omega_{3}$ time-domain response. (b) $\omega_{o} \approx \omega_{3}$ frequency-domain response.

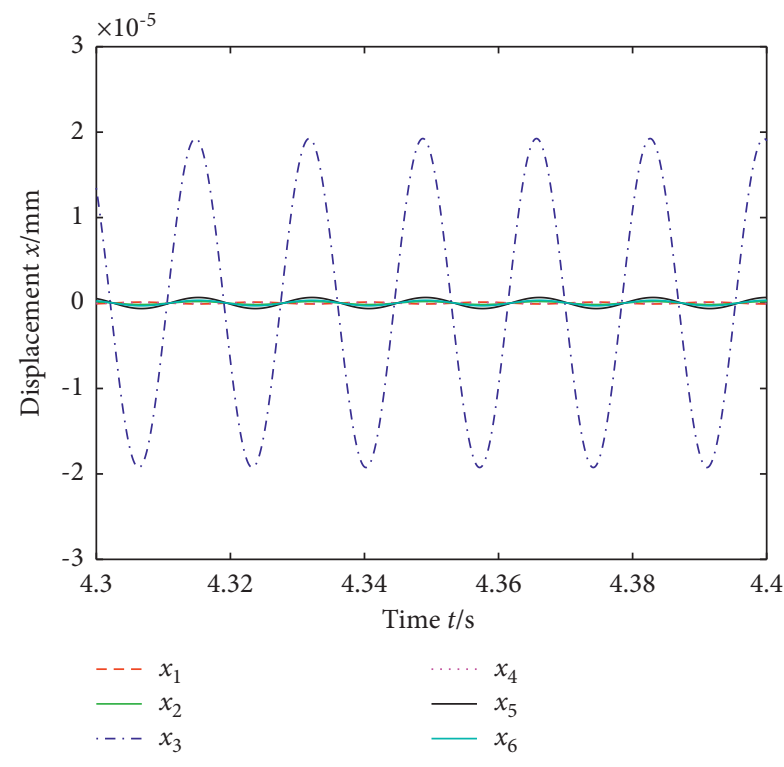

(a)

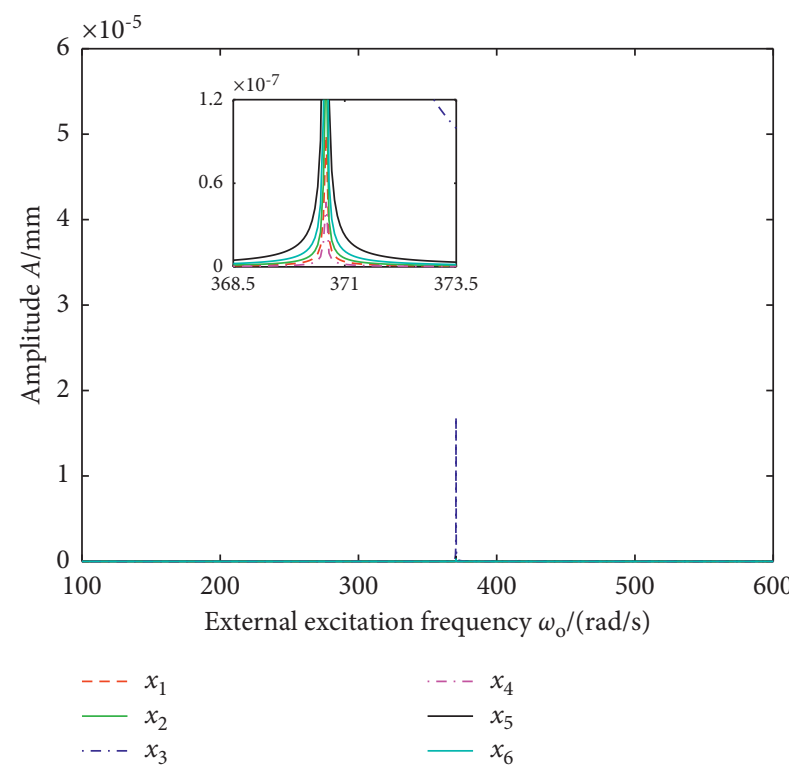

(b)

FIgURe 5: Main vibration response of the electro-hydraulic Stewart platform transmission system at $\omega_{o} \approx \omega_{4}$. (a) $\omega_{o} \approx \omega_{4}$ time-domain response. (b) $\omega_{o} \approx \omega_{4}$ frequency-domain response.

stable value of the first-order to the sixth-order natural angular frequency of the electro-hydraulic platform transmission system can be seen from Figures 14-19. With the change of position and pose, the natural frequency of the system changes and the main resonance response also changes. As can be seen from Figures 14(a)-19(a), while the external excitation frequency is close to the steady-state value of the natural frequency of the first to sixth order of the transmission system, the system will experience a relatively intense primary resonance with a maximum amplitude of $0.00002923 \mathrm{~mm}$. Compared with pose 1 , the modal amplitudes of the system are all increased. This indicates that, in the asymmetric pose, the connection between each leg is closer, the coupling is increased, and the degree of participation in vibration is increased. As can be 


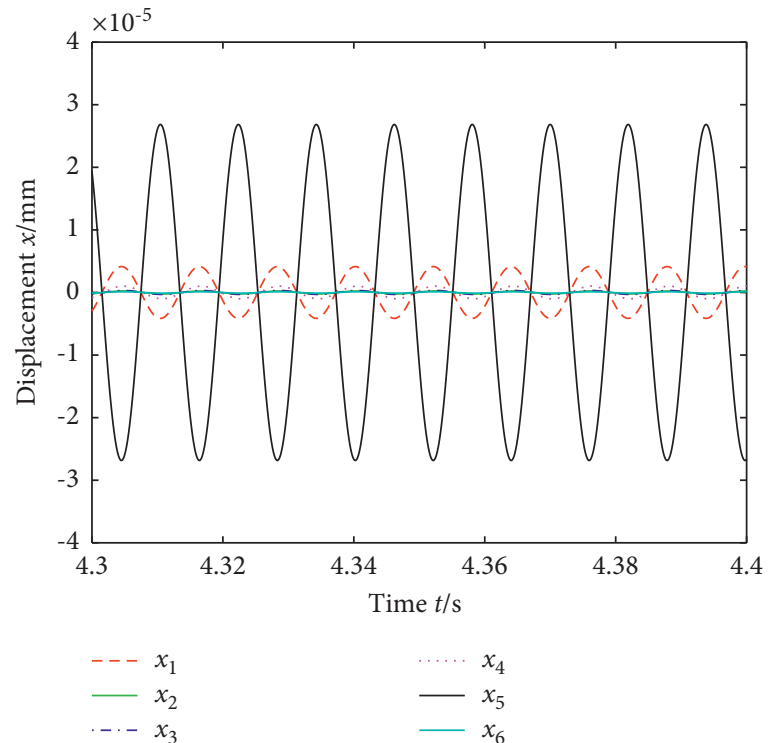

(a)

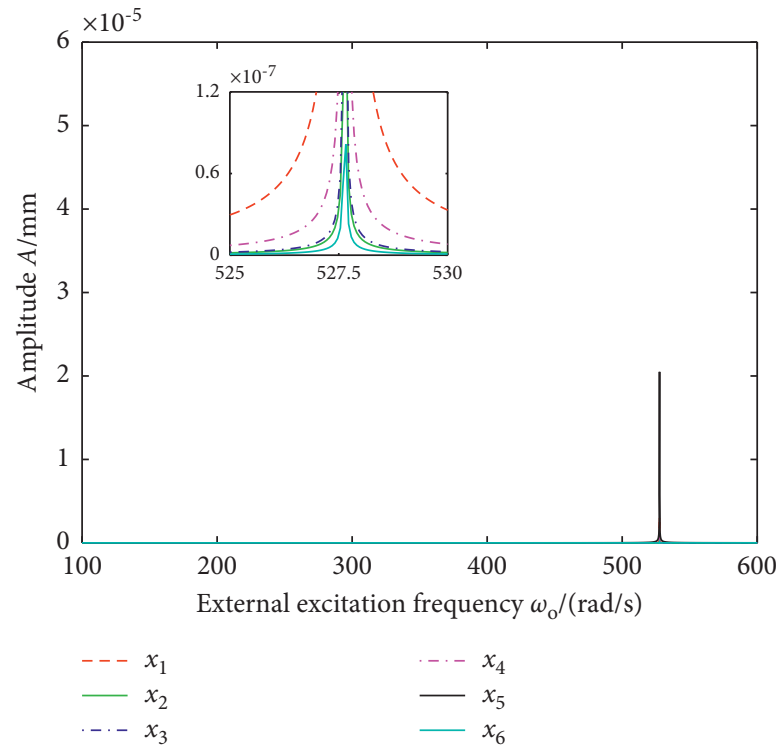

(b)

FiguRE 6: Main vibration response of the electro-hydraulic Stewart platform transmission system at $\omega_{o} \approx \omega_{5}$. (a) $\omega_{o} \approx \omega_{5}$ time-domain response. (b) $\omega_{o} \approx \omega_{5}$ frequency-domain response.

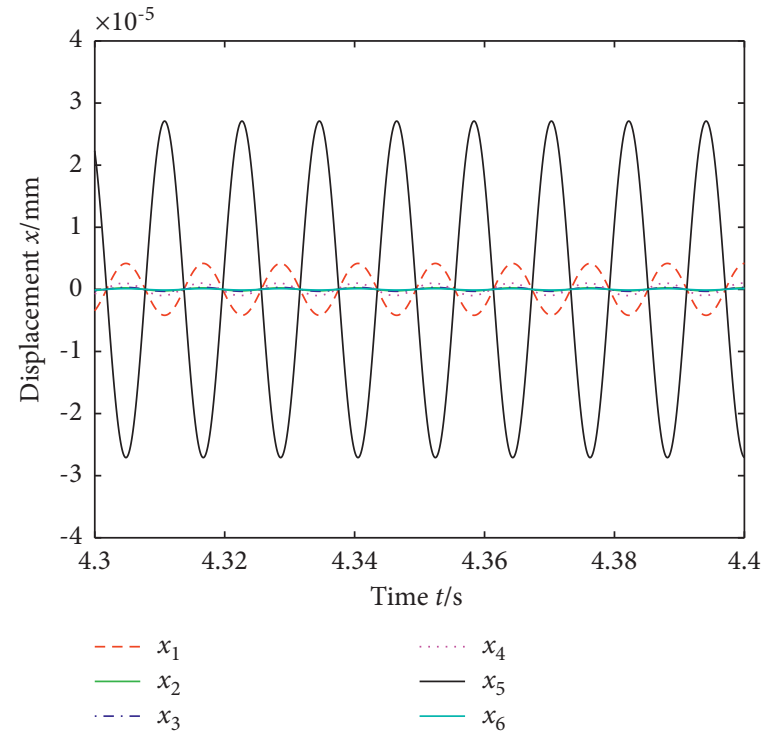

(a)

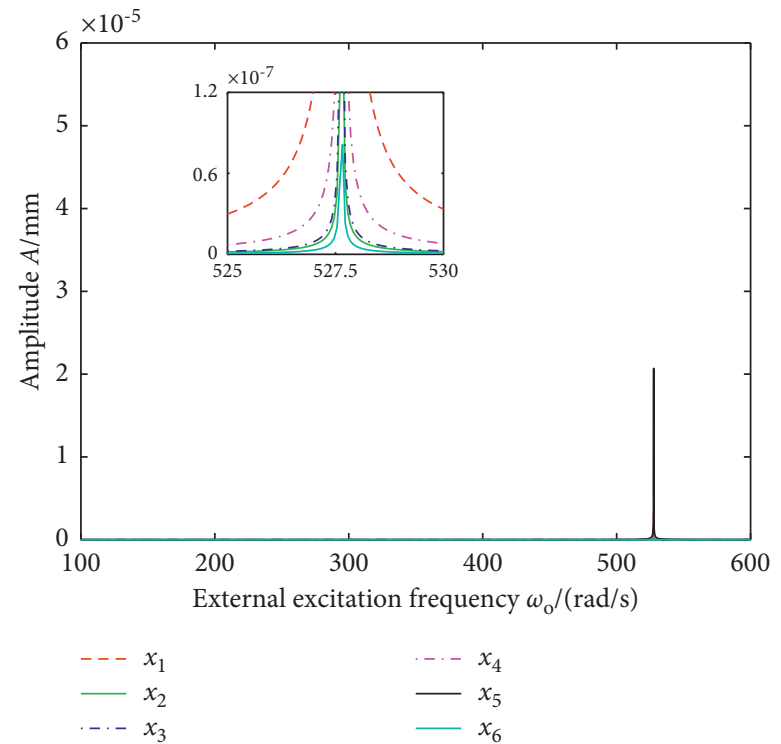

(b)

Figure 7: Main vibration response of the electro-hydraulic Stewart platform transmission system at $\omega_{o} \approx \omega_{6}$. (a) $\omega_{o} \approx \omega_{6}$ time-domain response. (b) $\omega_{o} \approx \omega_{6}$ frequency-domain response.

seen from Figures 14(b)-19(b), when the main resonance is generated in the system, there is a spike in the frequency-domain image, and the corresponding frequency of the peak corresponds to the approximate analytical formula of the main resonance of the system. Its peak corresponds to the external excitation frequency close to the natural frequency. This indicates that it plays a leading role in the main resonance of the system. In this case, the dominant frequency is the sum of the natural frequencies of each order and the fluctuation frequency of the equivalent stiffness of hydraulic oil, and other natural frequencies also reach their maximum amplitudes at the dominant frequency.

5.2.2. Combined Resonance Response. As can be seen from Figures 20(a)-25(a), when the external excitation frequency is close to the combination frequency of the stable value of 


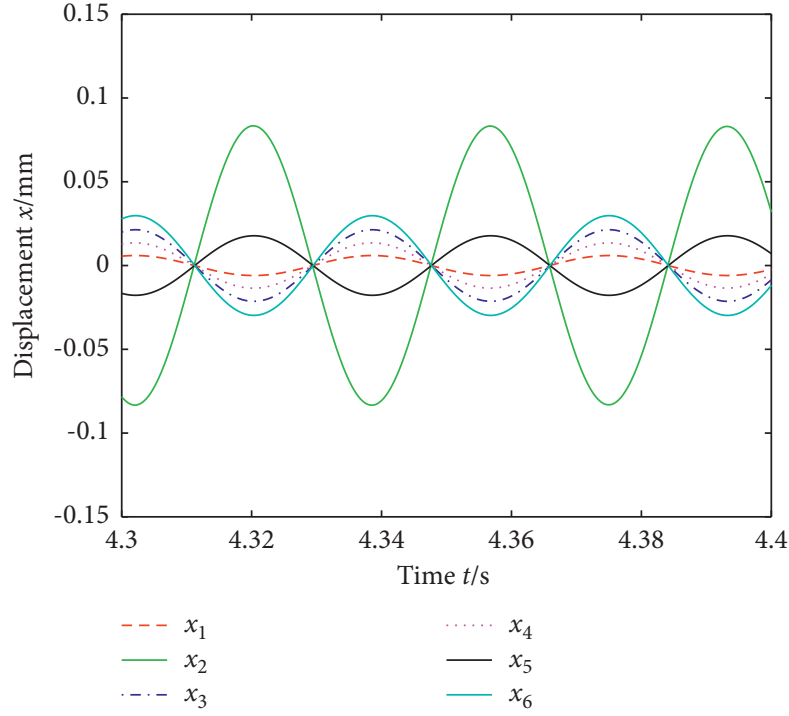

(a)

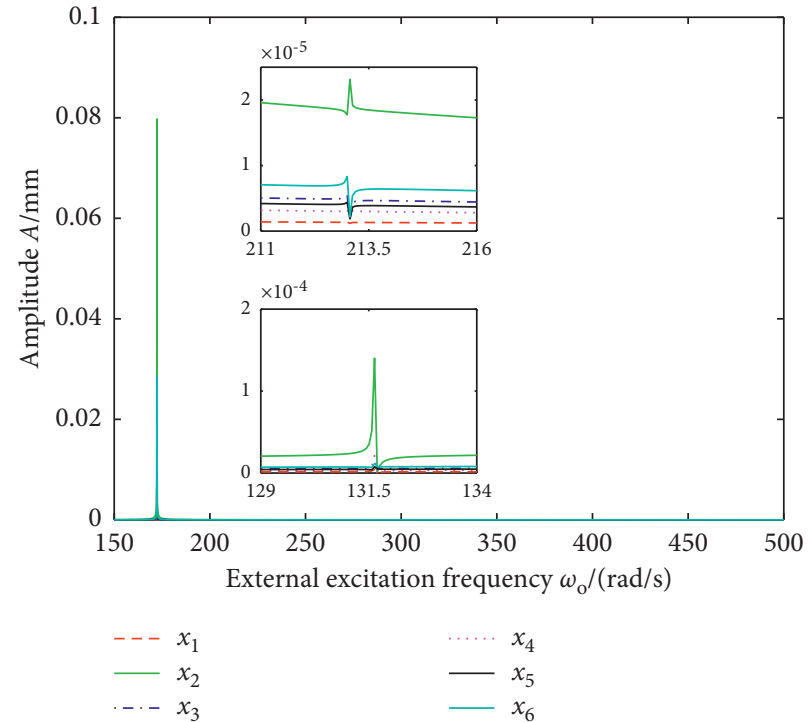

(b)

Figure 8: Combined vibration response of the electro-hydraulic Stewart platform transmission system at $\omega_{o} \approx \omega_{1}+\omega_{e}$. (a) $\omega_{o} \approx \omega_{1}+\omega_{e}$ timedomain response. (b) $\omega_{o} \approx \omega_{1}+\omega_{e}$ frequency-domain response.

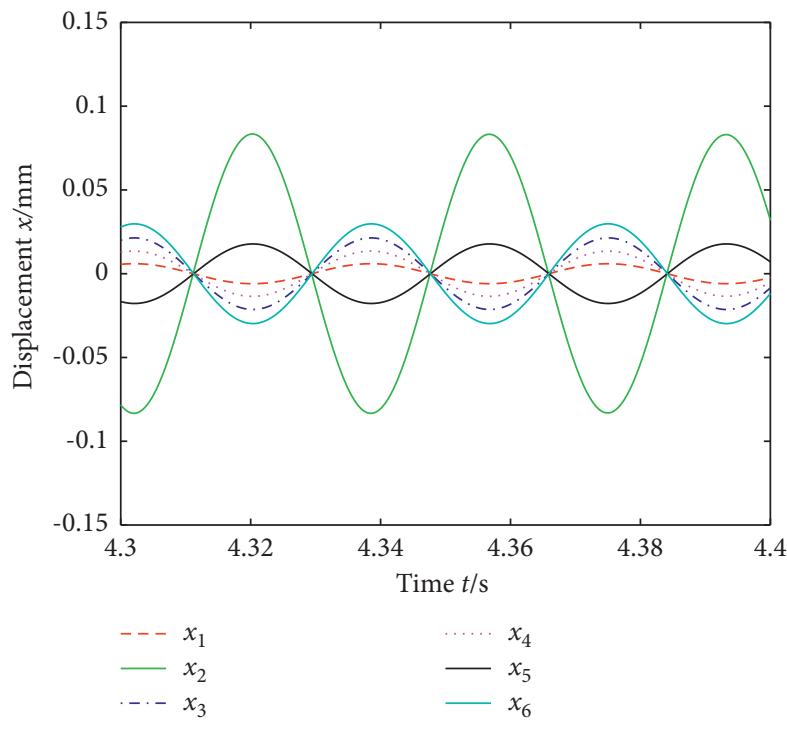

(a)

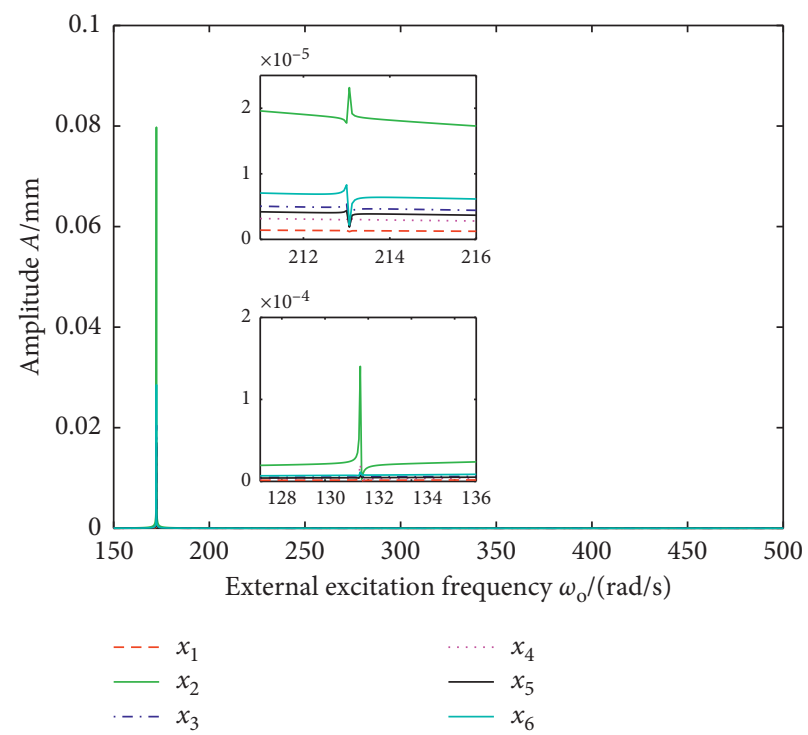

(b)

Figure 9: Combined vibration response of the electro-hydraulic Stewart platform transmission system at $\omega_{o} \approx \omega_{2}+\omega_{e}$. (a) $\omega_{o} \approx \omega_{2}+\omega_{e}$ timedomain response. (b) $\omega_{o} \approx \omega_{2}+\omega_{e}$ frequency-domain response.

the first to sixth order natural angular frequency of the electro-hydraulic platform transmission system and the fluctuation angular frequency of the equivalent stiffness of hydraulic oil, the combined resonance phenomenon occurs in the system and the amplitude is relatively severe. Compared with pose 1, the modal amplitudes of the system are all increased. This indicates that, in the asymmetric pose, the connection between each leg is closer, the coupling is increased, and the degree of participation in vibration is increased. This indicates that, in the asymmetric posture, the connection between each leg is more close, and the degree of participation in vibration is increased. As can be seen from Figures 20(b)-25(b), when the system produces combined resonance, there are three spikes in the frequencydomain image, and the corresponding frequencies of each spike correspond to the approximate analytical formula of the system's main resonance one by one. Among them, the peak corresponding to the combined frequency $\omega_{o} \approx \omega_{i}+\omega_{e}$ of the stable value of the natural angular frequency of each order of the system and the fluctuation 


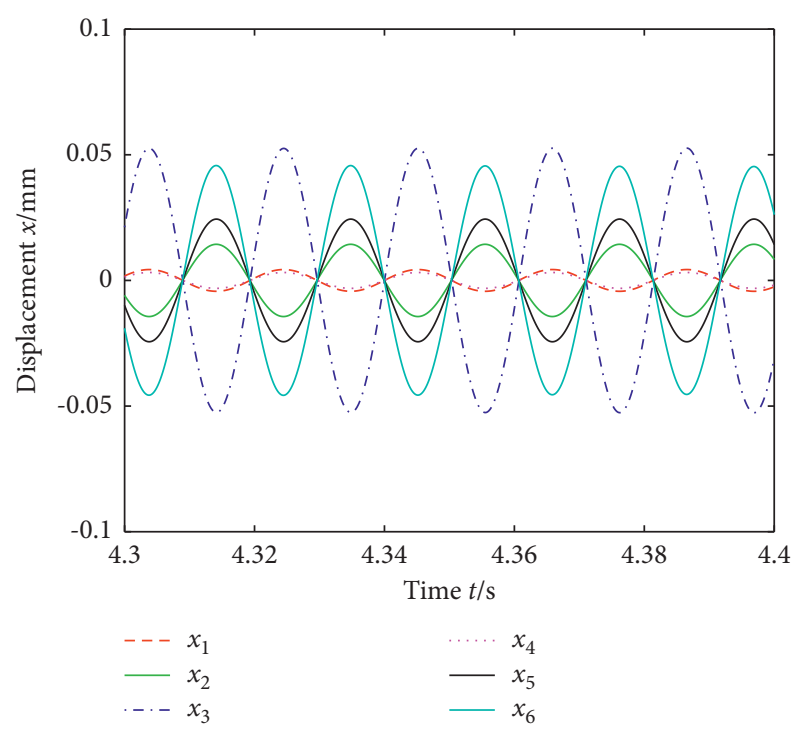

(a)

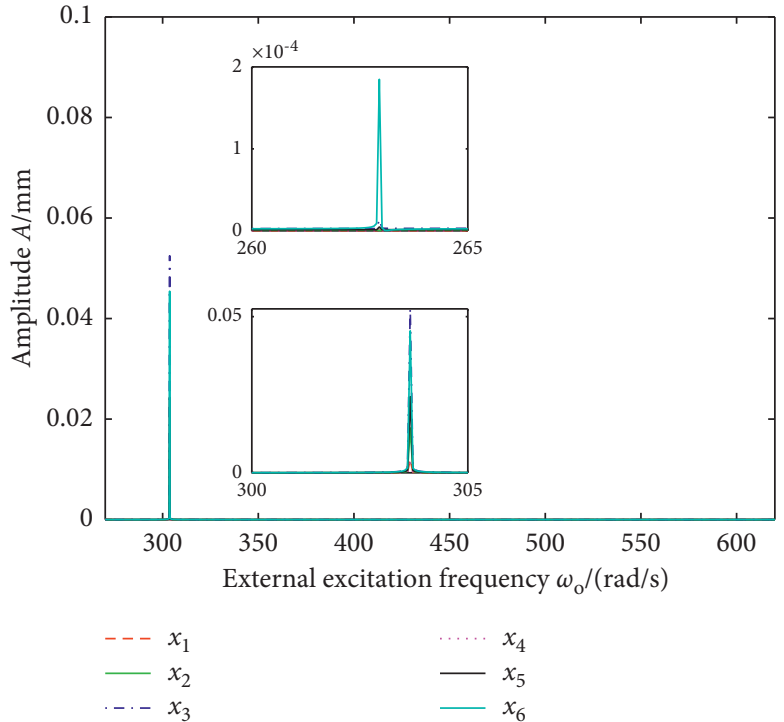

(b)

Figure 10: Combined vibration response of the electro-hydraulic Stewart platform transmission system at $\omega_{o} \approx \omega_{3}+\omega_{e}$. (a) $\omega_{o} \approx \omega_{3}+\omega_{e}$ time-domain response. (b) $\omega_{o} \approx \omega_{3}+\omega_{e}$ frequency-domain response.

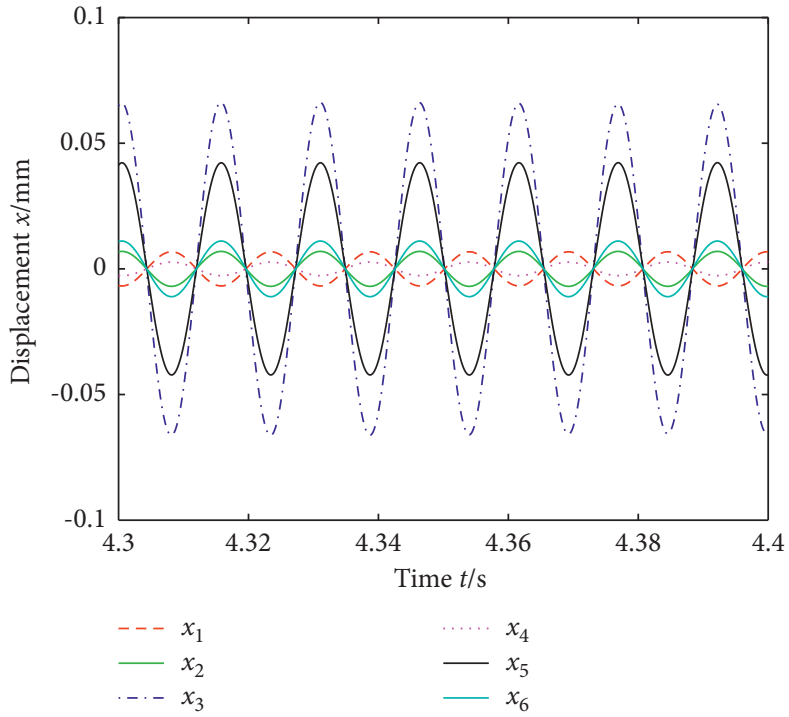

(a)

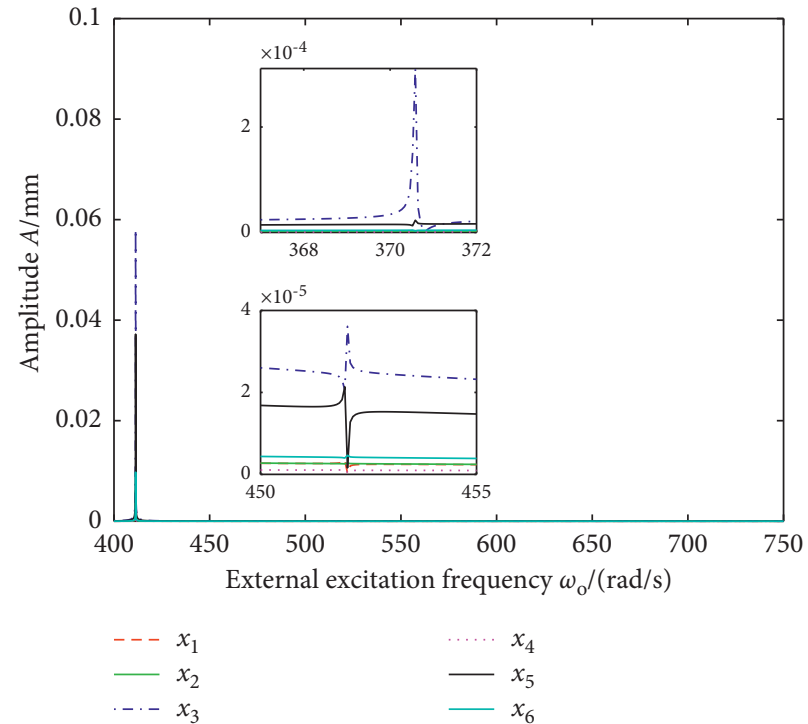

(b)

FIgURe 11: Combined vibration response of the electro-hydraulic Stewart platform transmission system at $\omega_{o} \approx \omega_{4}+\omega_{e}$. (a) $\omega_{o} \approx \omega_{4}+\omega_{e}$ time-domain response. (b) $\omega_{o} \approx \omega_{4}+\omega_{e}$ frequency-domain response.

angular frequency of the equivalent stiffness of hydraulic oil is the highest. It shows that it plays a leading role in the combined resonance of the system and the other frequencies play a regulating role. There is a lower peak on the left and right side of the peak corresponding to the combined frequencies, with the left corresponding frequency $\omega_{o} \approx \omega_{i}$ and the right corresponding frequency $\omega_{o} \approx \omega_{i}+2 \omega_{e}(i=1,2, \ldots, 6)$. 


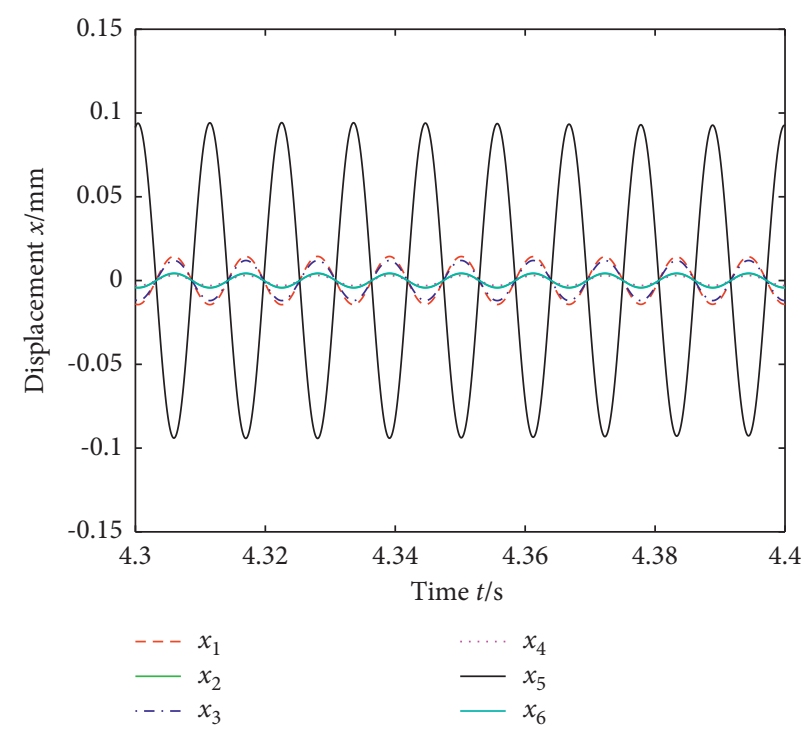

(a)

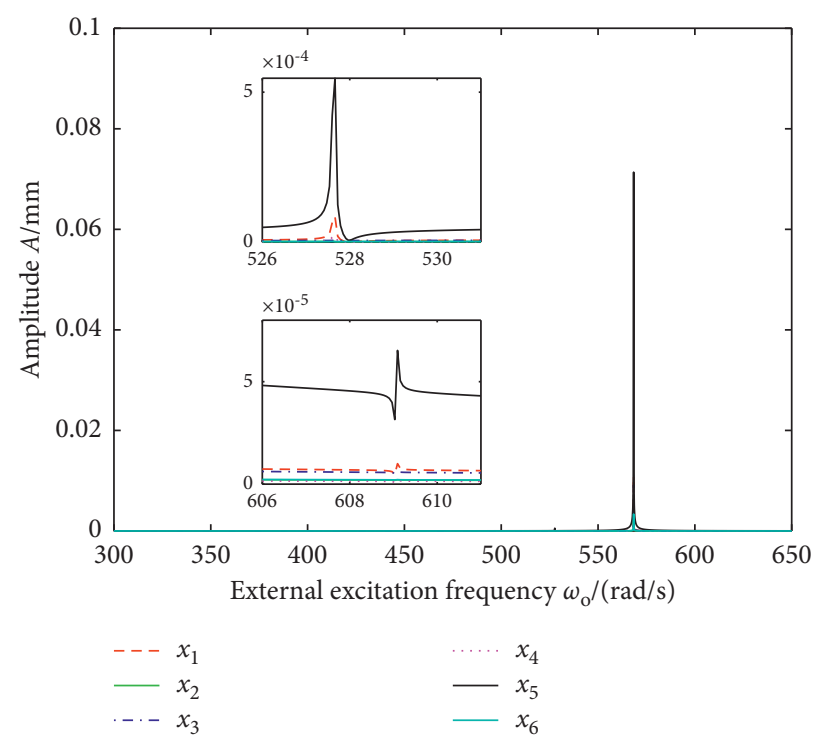

(b)

FIGURe 12: Combined vibration response of the electro-hydraulic Stewart platform transmission system at $\omega_{o} \approx \omega_{5}+\omega_{e}$. (a) $\omega_{o} \approx \omega_{5}+\omega_{e}$ time-domain response. (b) $\omega_{o} \approx \omega_{5}+\omega_{e}$ frequency-domain response.

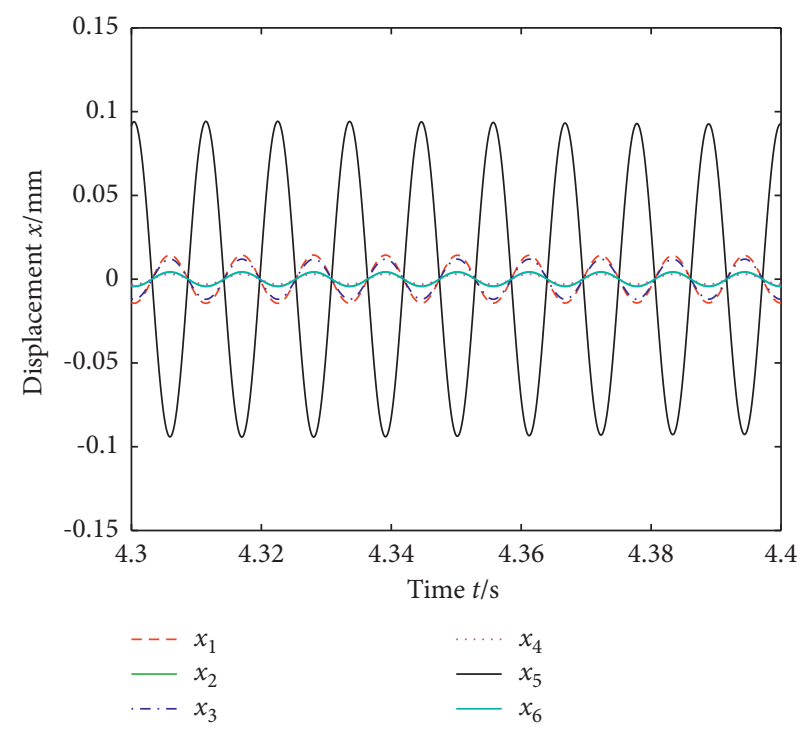

(a)

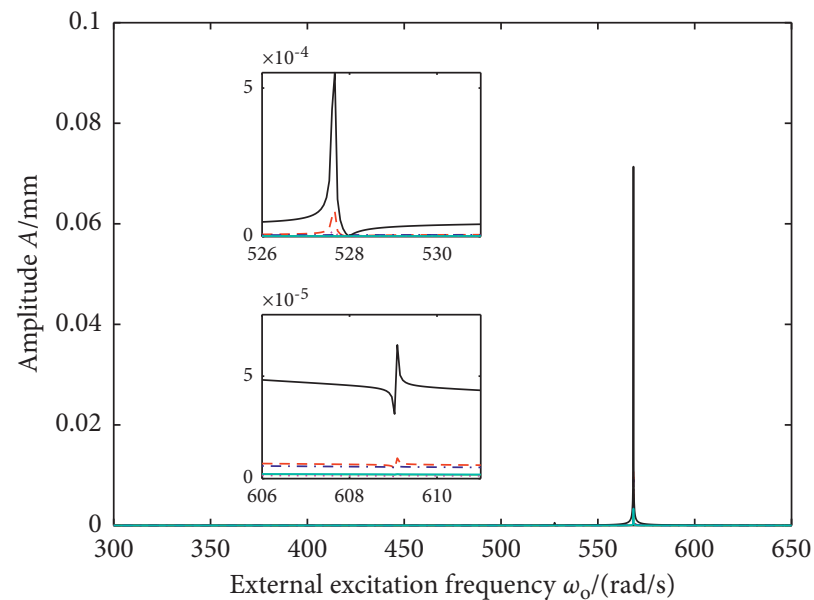

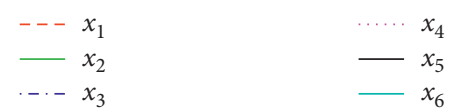

(b)

FIGURe 13: Combined vibration response of the electro-hydraulic Stewart platform transmission system at $\omega_{o} \approx \omega_{6}+\omega_{e}$. (a) $\omega_{o} \approx \omega_{6}+\omega_{e}$ time-domain response. (b) $\omega_{o} \approx \omega_{6}+\omega_{e}$ frequency-domain response. 
TABLE 3: Calculation parameters related to vibration of 6-DOF platform transmission system parameters.

\begin{tabular}{|c|c|}
\hline Name & Value \\
\hline Steady-state value of the first-order natural angular frequency $\omega_{1}\left(\mathrm{rad} \cdot \mathrm{s}^{-1}\right)$ & 111.1024 \\
\hline Steady-state value of the second-order natural angular frequency $\omega_{2}\left(\mathrm{rad} \cdot \mathrm{s}^{-1}\right)$ & 124.4199 \\
\hline Steady-state value of the third-order natural angular frequency $\omega_{3}\left(\mathrm{rad} \cdot \mathrm{s}^{-1}\right)$ & 235.3889 \\
\hline Steady-state value of the fourth-order natural angular frequency $\omega_{4}\left(\mathrm{rad} \cdot \mathrm{s}^{-1}\right)$ & 375.2415 \\
\hline Steady-state value of the fifth-order natural angular frequency $\omega_{5}\left(\mathrm{rad} \cdot \mathrm{s}^{-1}\right)$ & 505.6999 \\
\hline Steady-state value of the sixth-order natural angular frequency $\omega_{6}\left(\mathrm{rad} \cdot \mathrm{s}^{-1}\right)$ & 518.4187 \\
\hline Fluctuation angular frequency of equivalent stiffness of fluid element $\omega_{e}\left(\mathrm{rad} \cdot \mathrm{s}^{-1}\right)$ & 40.7150 \\
\hline Amplitude of external excitation $F_{0}(\mathrm{~N})$ & {$\left[\begin{array}{llllll}20 & 20 & 20 & 20 & 20 & 20\end{array}\right]^{T}$} \\
\hline Structural damping $C / N \cdot(\mathrm{m} / \mathrm{s})$ & {$\left[\begin{array}{llllll}20 & 20 & 20 & 20 & 20 & 20\end{array}\right]^{T}$} \\
\hline
\end{tabular}

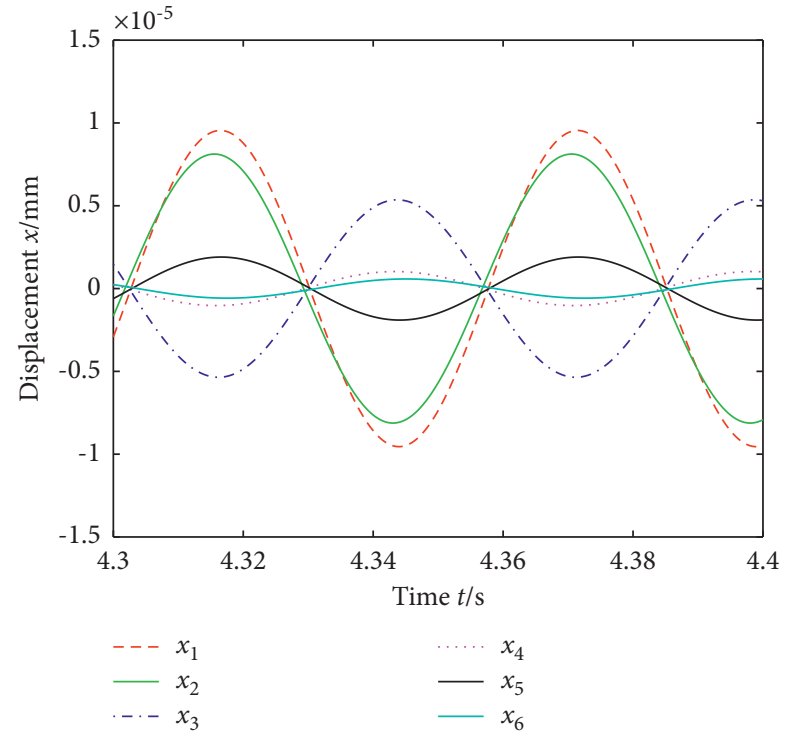

(a)

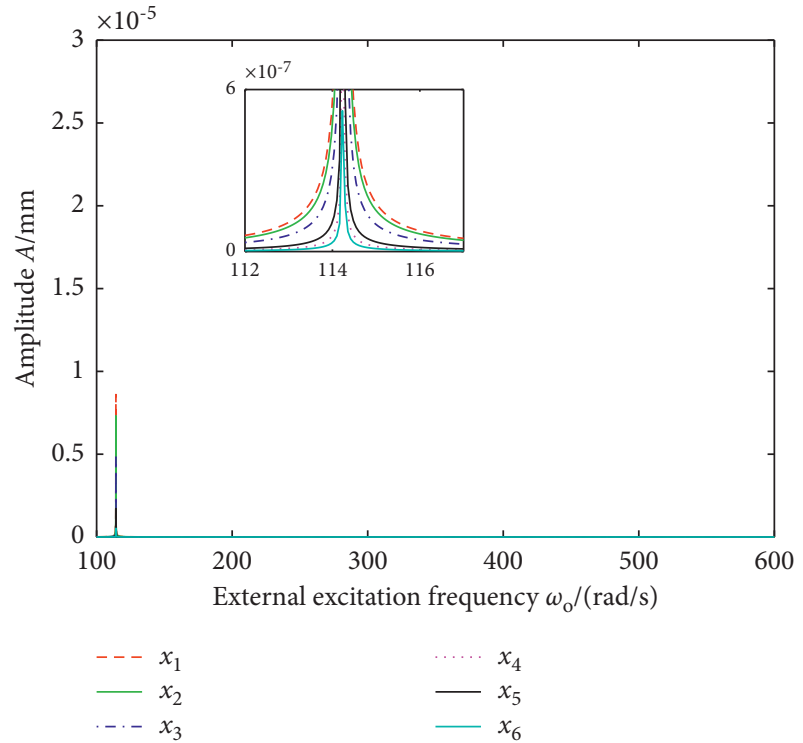

(b)

FIGURE 14: Main vibration response of the electro-hydraulic Stewart platform transmission system at $\omega_{o} \approx \omega_{1}$. (a) $\omega_{o} \approx \omega_{1}$ time-domain response. (b) $\omega_{o} \approx \omega_{1}$ frequency-domain response.

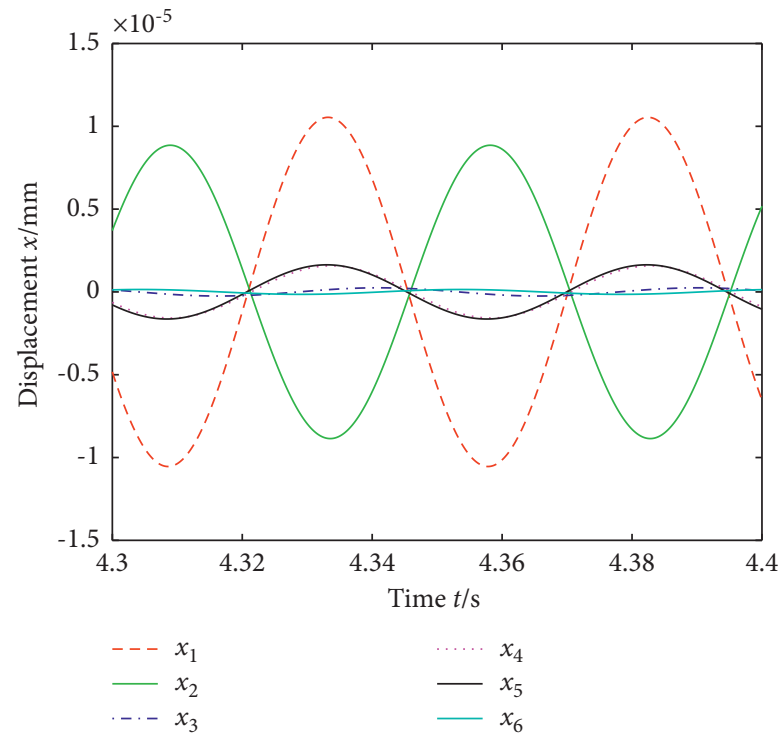

(a)

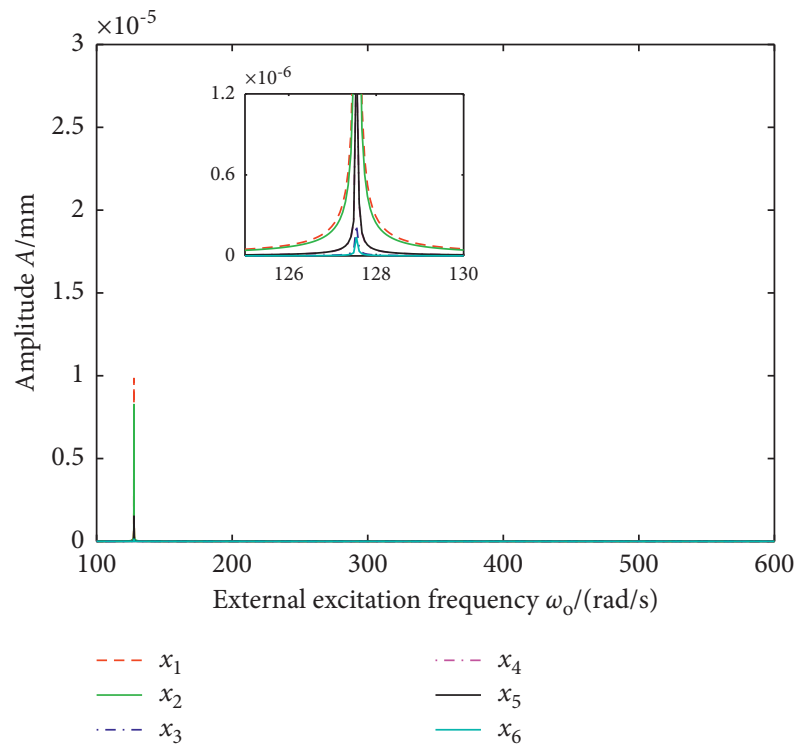

(b)

FIgURe 15: Main vibration response of the electro-hydraulic Stewart platform transmission system at $\omega_{o} \approx \omega_{2}$. (a) $\omega_{o} \approx \omega_{2}$ time-domain response. (b) $\omega_{o} \approx \omega_{2}$ frequency-domain response. 


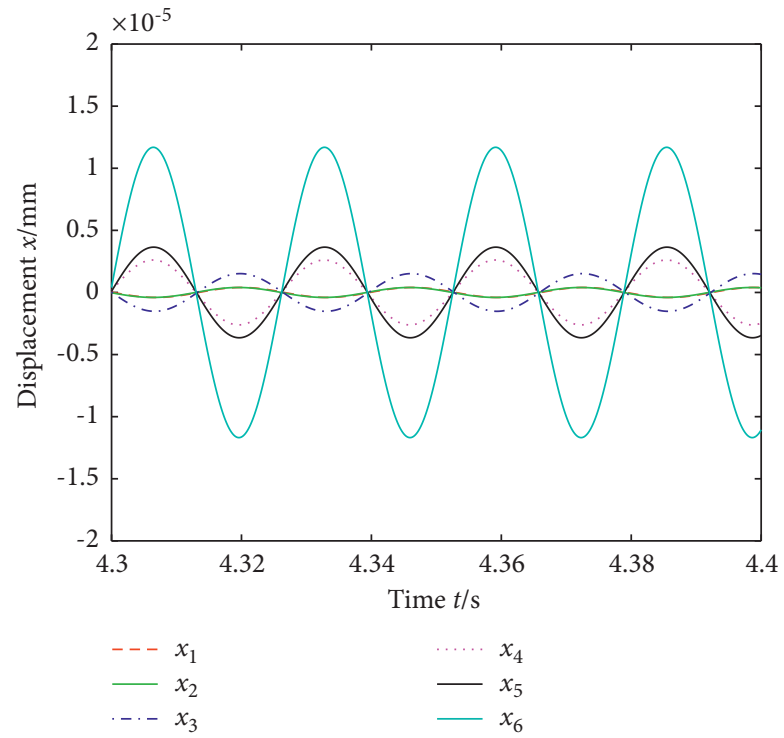

(a)

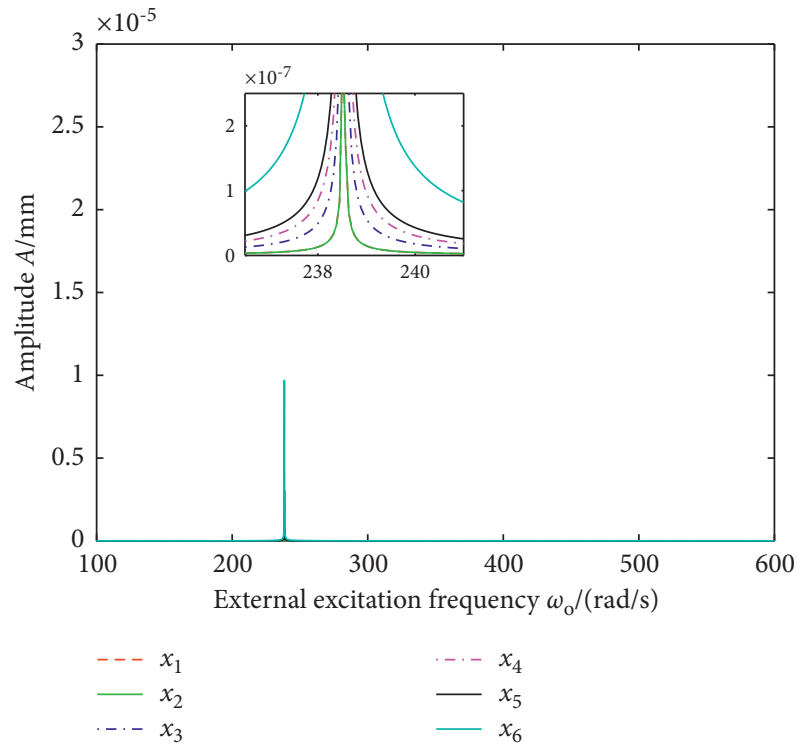

(b)

FIgURe 16: Main vibration response of the electro-hydraulic Stewart platform transmission system at $\omega_{o} \approx \omega_{3}$. (a) $\omega_{o} \approx \omega_{3}$ time-domain response. (b) $\omega_{o} \approx \omega_{3}$ frequency-domain response.

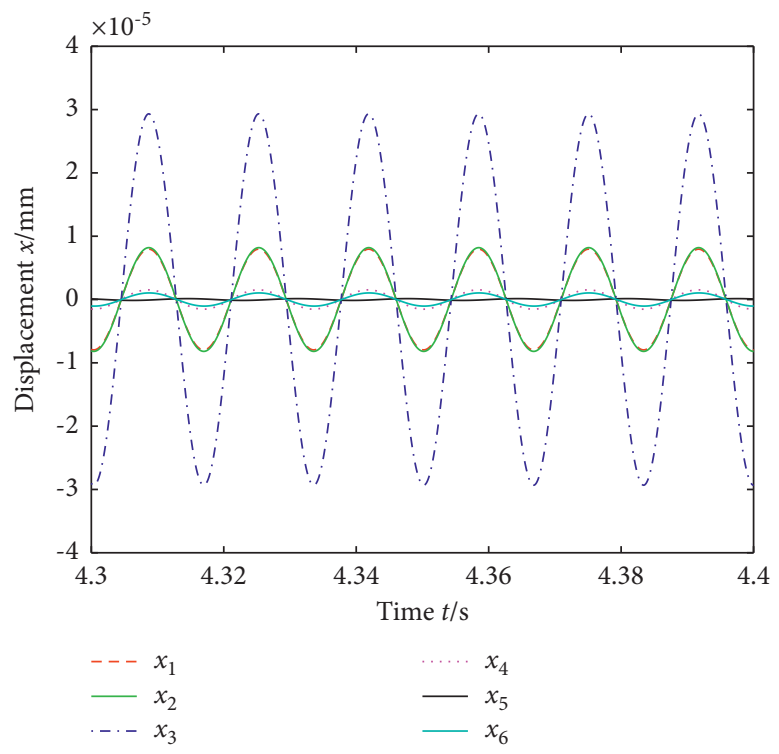

(a)

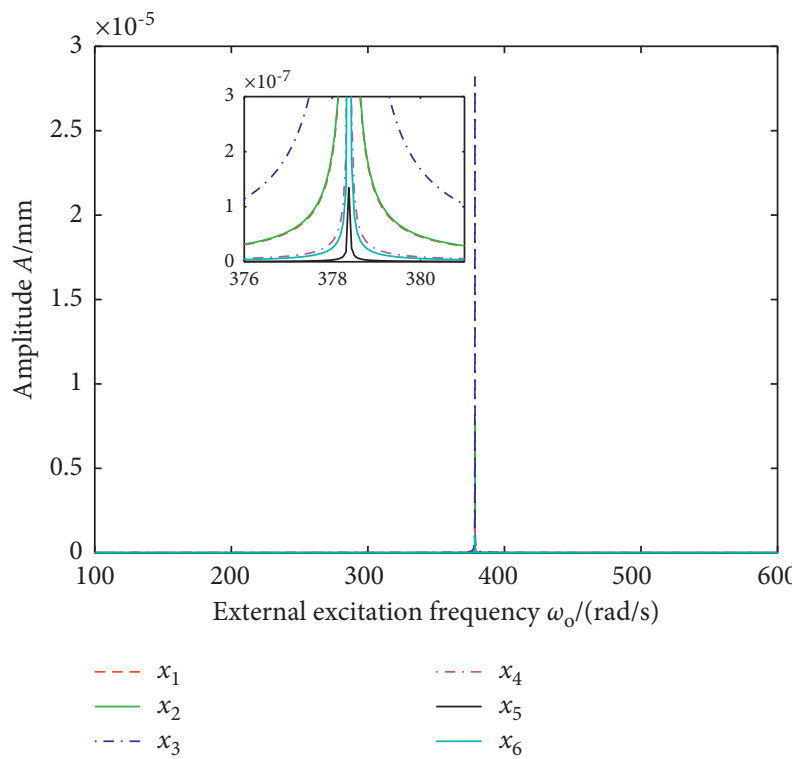

(b)

FIgURe 17: Main vibration response of the electro-hydraulic Stewart platform transmission system at $\omega_{o} \approx \omega_{4}$. (a) $\omega_{o} \approx \omega_{4}$ time-domain response. (b) $\omega_{o} \approx \omega_{4}$ frequency-domain response. 


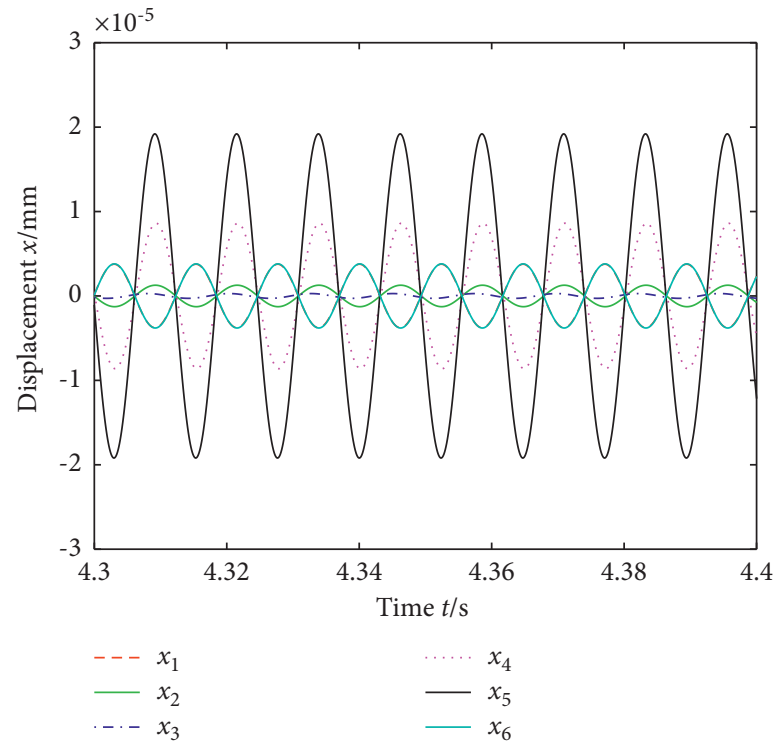

(a)

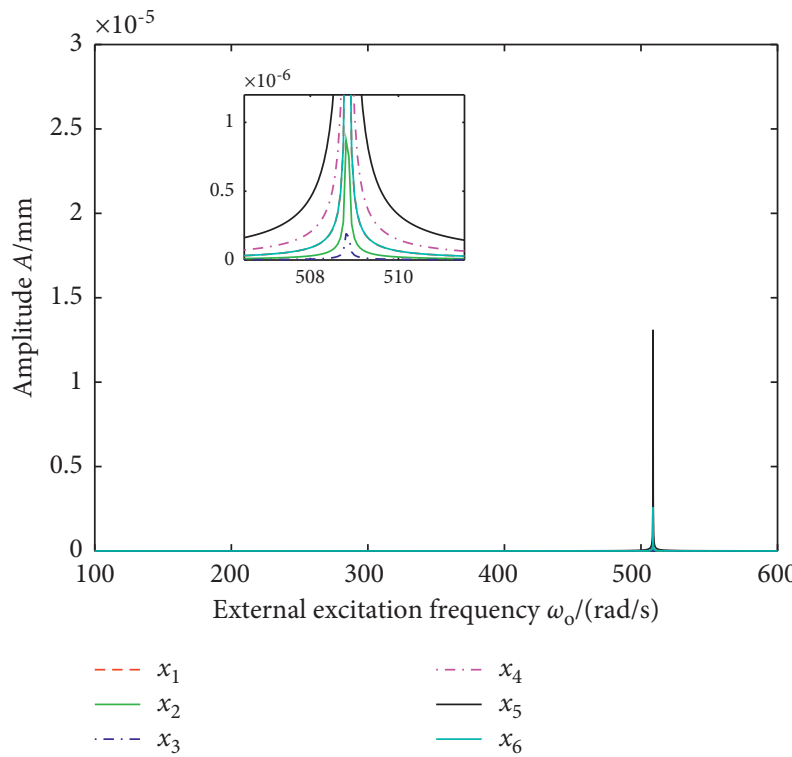

(b)

FIGURE 18: Main vibration response of the electro-hydraulic Stewart platform transmission system at $\omega_{o} \approx \omega_{5}$. (a) $\omega_{o} \approx \omega_{5}$ time-domain response. (b) $\omega_{o} \approx \omega_{5}$ frequency-domain response.

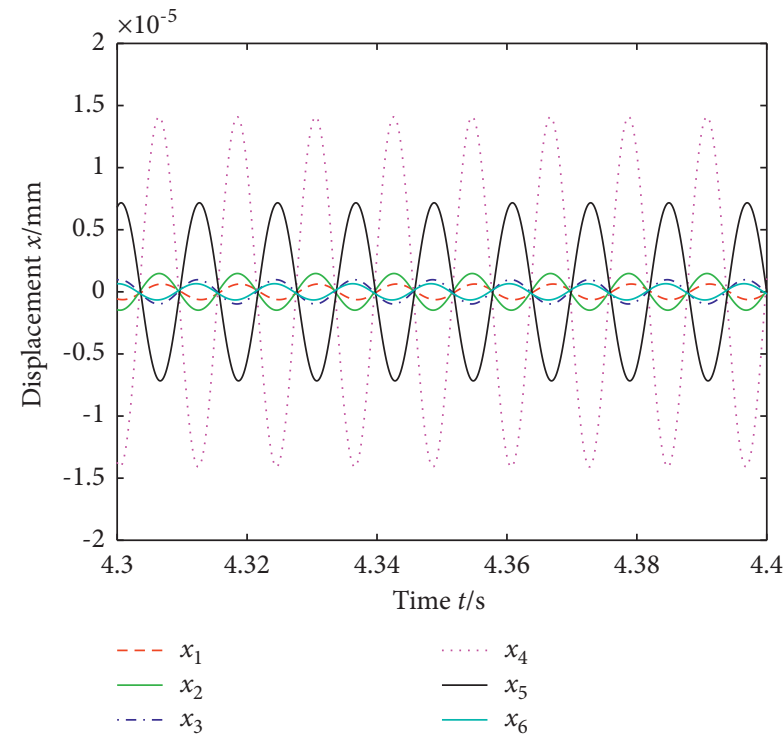

(a)

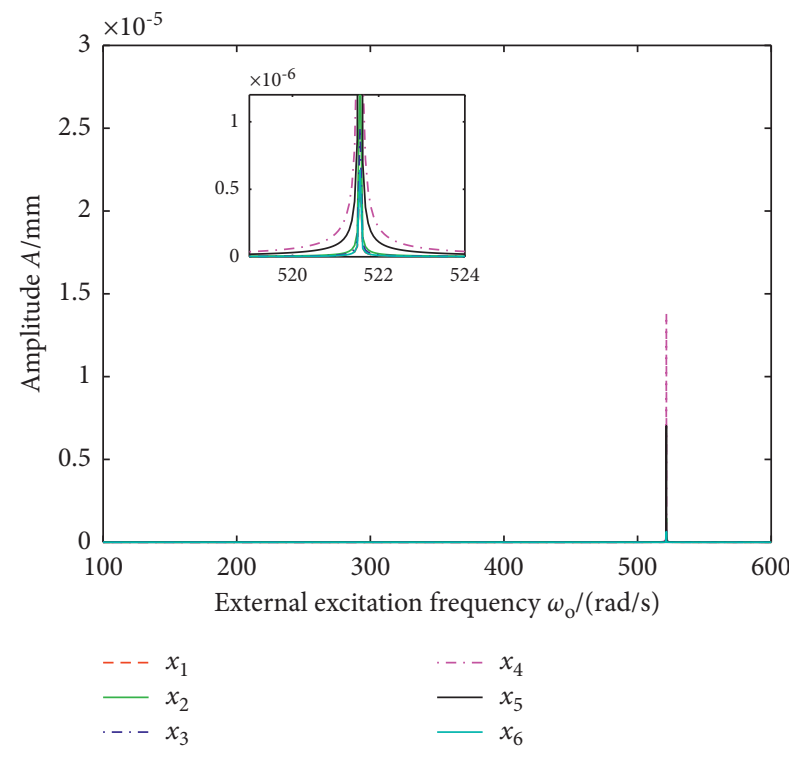

(b)

FIGURE 19: Main vibration response of the electro-hydraulic Stewart platform transmission system at $\omega_{0} \approx \omega_{6}$. (a) $\omega_{o} \approx \omega_{6}$ time-domain response. (b) $\omega_{o} \approx \omega_{6}$ frequency-domain response. 


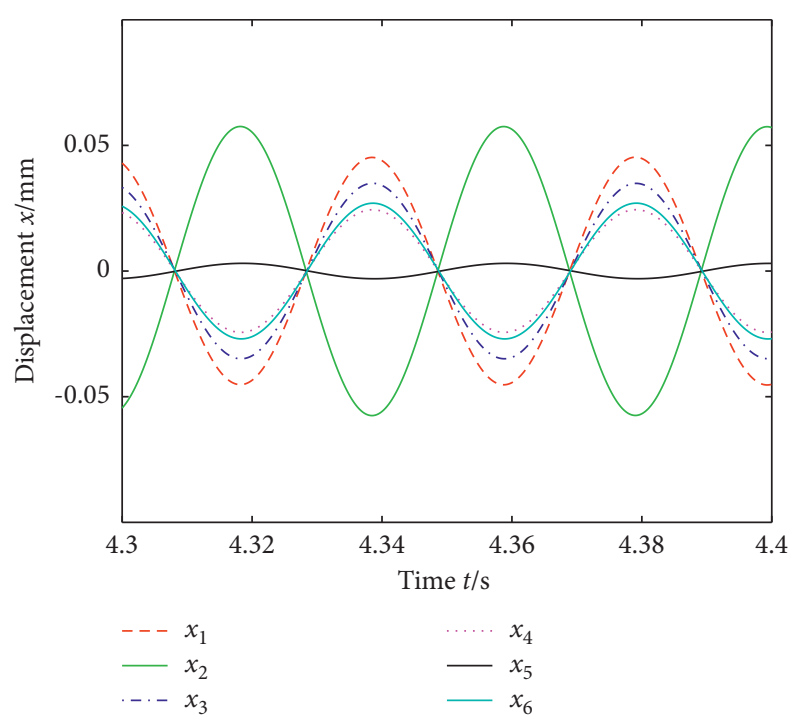

(a)

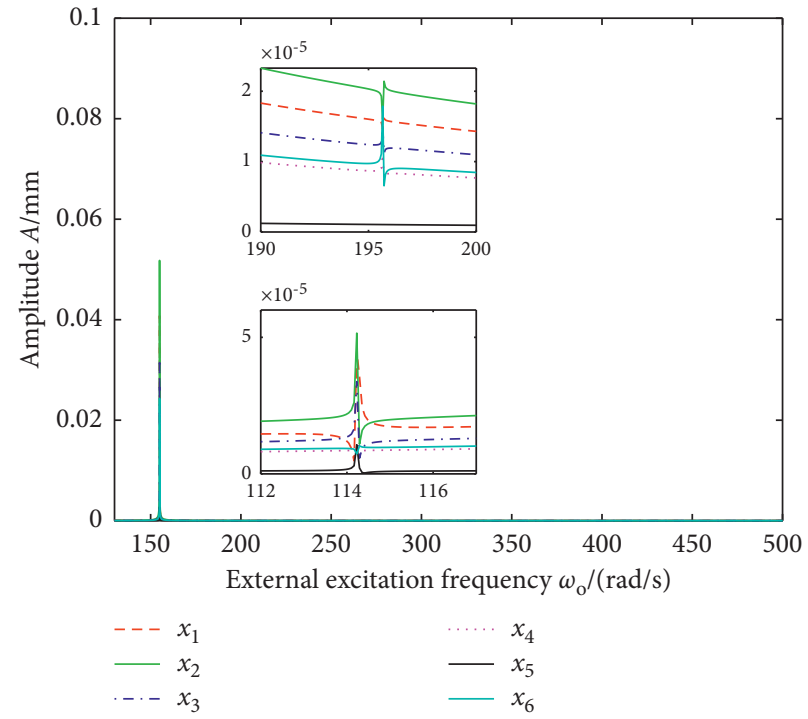

(b)

Figure 20: Combined vibration response of the electro-hydraulic Stewart platform transmission system at $\omega_{o} \approx \omega_{1}+\omega_{e}$. (a) $\omega_{o} \approx \omega_{1}+\omega_{e}$ time-domain response. (b) $\omega_{o} \approx \omega_{1}+\omega_{e}$ frequency-domain response.

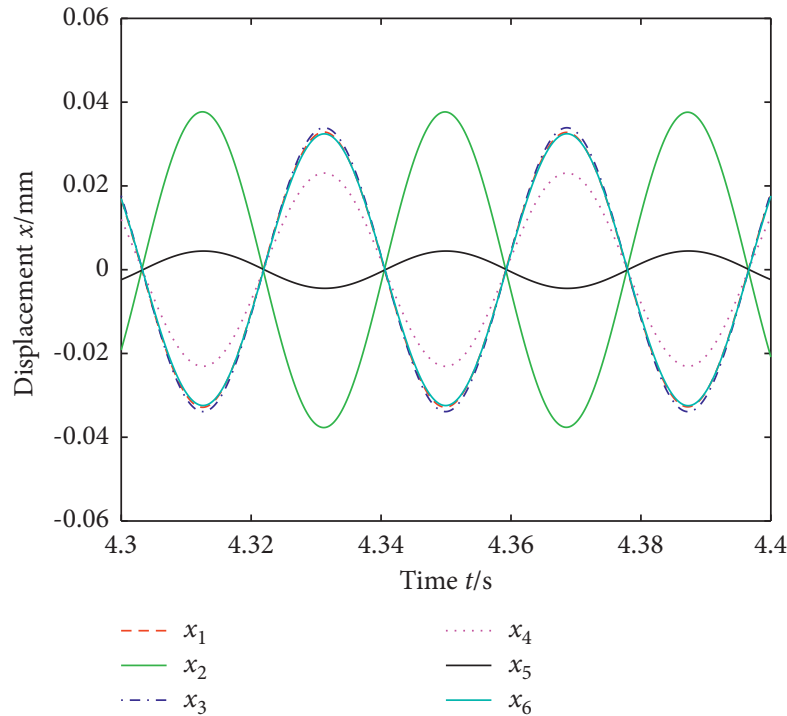

(a)

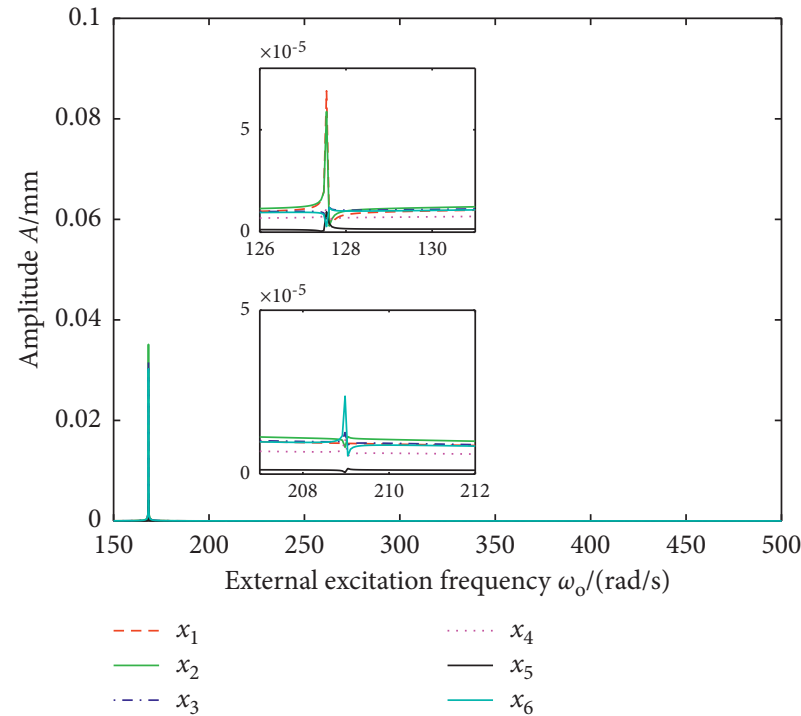

(b)

FiguRE 21: Combined vibration response of the electro-hydraulic Stewart platform transmission system at $\omega_{o} \approx \omega_{2}+\omega_{e}$. (a) $\omega_{o} \approx \omega_{2}+\omega_{e}$ time-domain response. (b) $\omega_{o} \approx \omega_{2}+\omega_{e}$ frequency-domain response. 


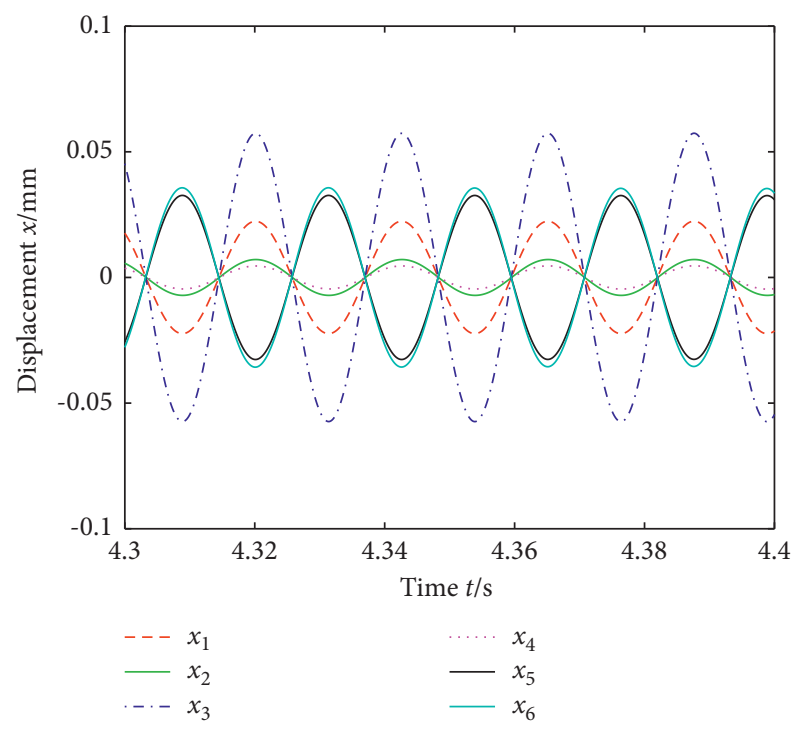

(a)

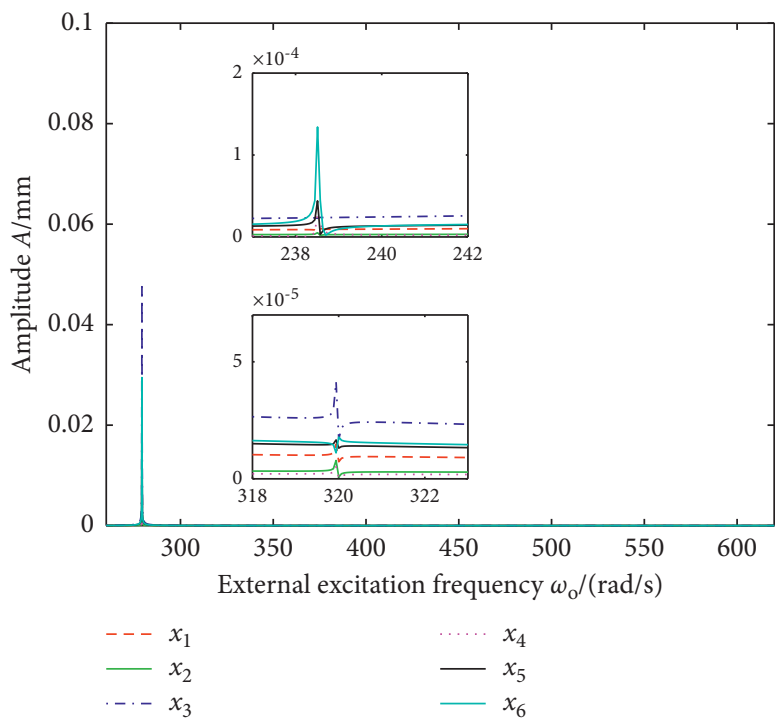

(b)

FIGURe 22: Combined vibration response of the electro-hydraulic Stewart platform transmission system at $\omega_{o} \approx \omega_{3}+\omega_{e}$. (a) $\omega_{o} \approx \omega_{3}+\omega_{e}$ time-domain response. (b) $\omega_{o} \approx \omega_{3}+\omega_{e}$ frequency-domain response.

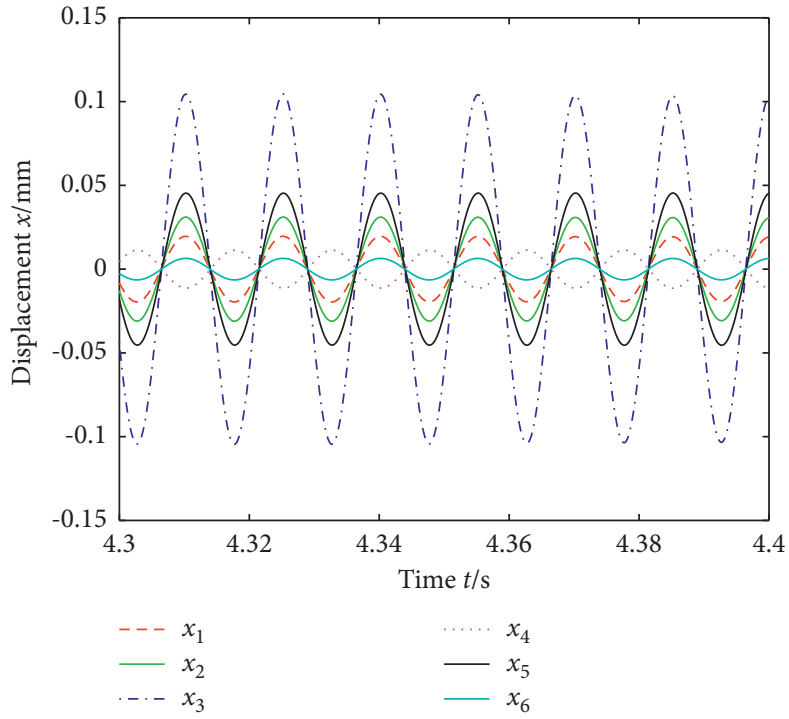

(a)

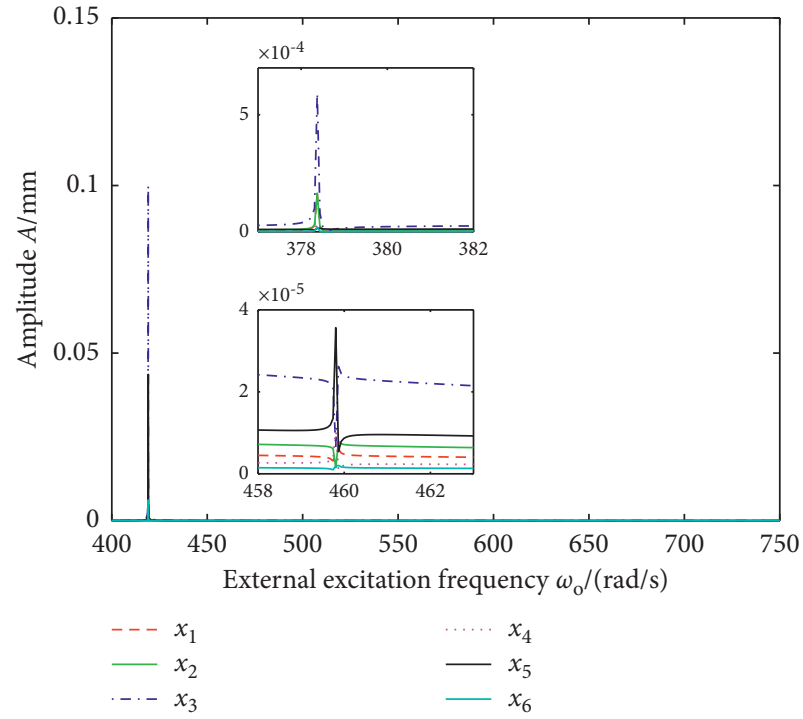

(b)

Figure 23: Combined vibration response of the electro-hydraulic Stewart platform transmission system at $\omega_{o} \approx \omega_{4}+\omega_{e}$. (a) $\omega_{o} \approx \omega_{4}+\omega_{e}$ time-domain response. (b) $\omega_{o} \approx \omega_{4}+\omega_{e}$ frequency-domain response. 


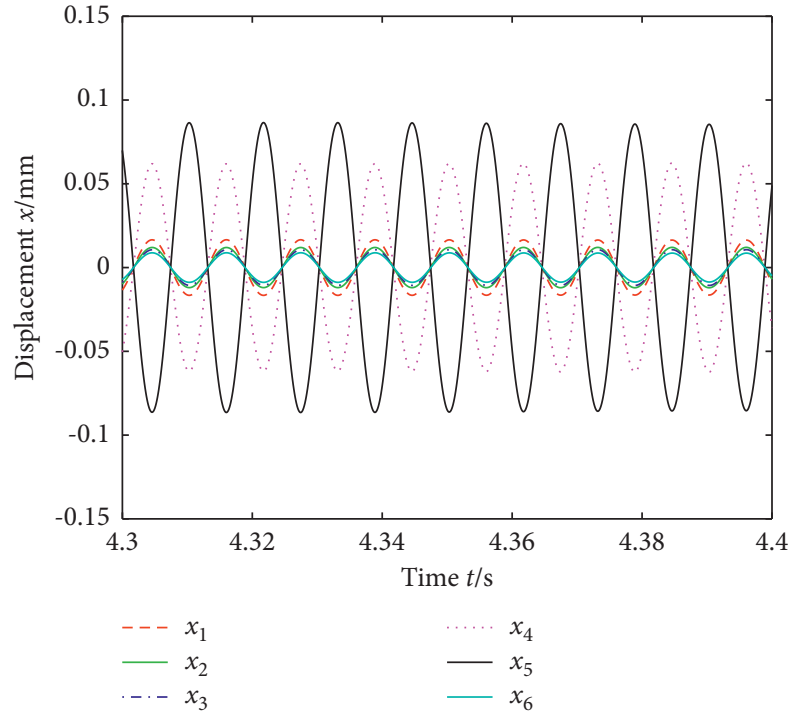

(a)

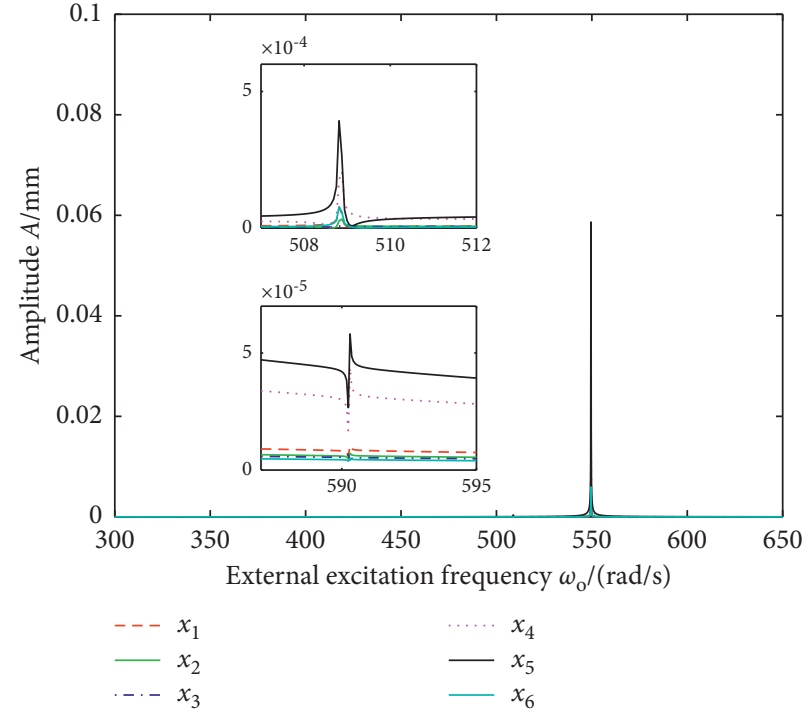

(b)

Figure 24: Combined vibration response of the electro-hydraulic Stewart platform transmission system at $\omega_{o} \approx \omega_{5}+\omega_{e}$. (a) $\omega_{o} \approx \omega_{5}+\omega_{e}$ time-domain response. (b) $\omega_{o} \approx \omega_{5}+\omega_{e}$ frequency-domain response.

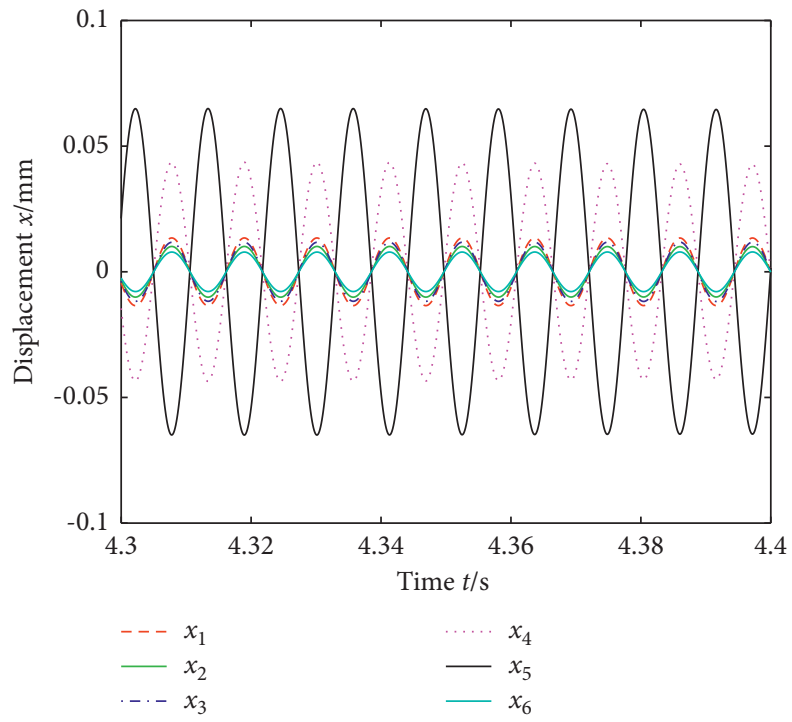

(a)

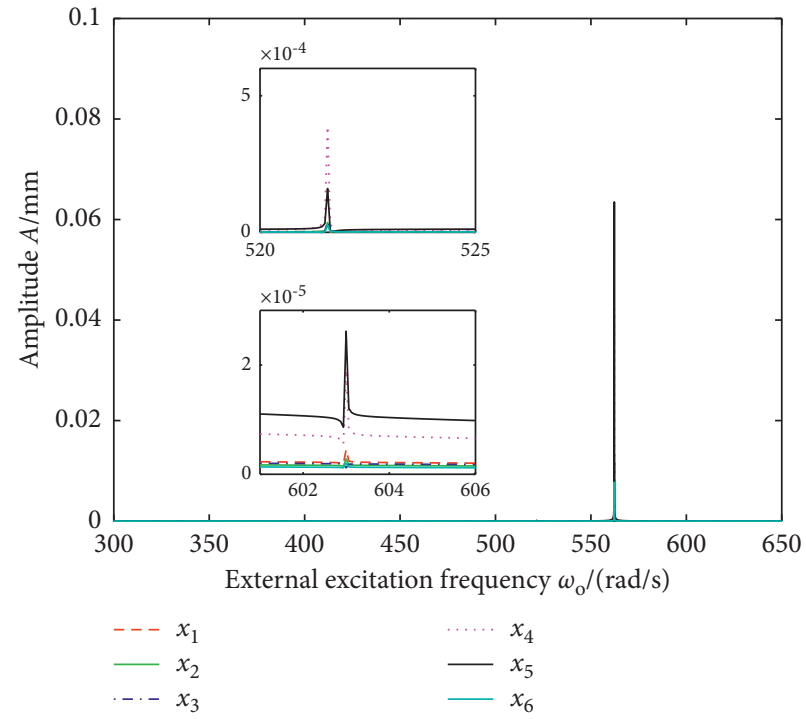

(b)

Figure 25: Combined vibration response of the electro-hydraulic Stewart platform transmission system at $\omega_{o} \approx \omega_{6}+\omega_{e}$. (a) $\omega_{o} \approx \omega_{6}+\omega_{e}$ time-domain response. (b) $\omega_{o} \approx \omega_{6}+\omega_{e}$ frequency-domain response.

\section{Conclusion}

In this paper, the parametric vibration dynamics equation of the Stewart 6-DOF platform is established. Based on the multiscale method, the theoretical formulas of the main resonance and the combined resonance response of the system are derived, and the parametric vibration response law of the system is analyzed. Its conclusions are as follows:

(1) The response characteristics of the main resonance response of the electro-hydraulic Stewart 6-DOF platform transmission system are mainly determined by the modal characteristics of the system; the combined resonance response of the transmission system is more complex. The natural frequencies of each order are the dominant frequencies, and the combined frequencies play a regulating role in the response.

(2) Through the platform of two different pose parameter vibration analysis, the platform position in the asymmetrical position compared with the symmetric position, the time-domain response of the 
system, and the frequency response analysis is more close to the actual situation; when the external excitation frequency close to the system natural frequency system of six order modal vibration amplitude is higher, each leg of the system participate in the higher degree of vibration with enhanced coupling.

The results provide a theoretical basis for the resonance phenomenon of the electro-hydraulic Stewart platform. It provides theoretical support for subsequent dynamic optimization and reliability analysis. Through analysis, it is suggested that the platform should be installed with a vibration absorber to reduce the excitation frequency only at a specific frequency to avoid resonance.

\section{Data Availability}

The data used to support the findings of this study are included within the article.

\section{Conflicts of Interest}

The authors declare that they have no conflicts of interest.

\section{Acknowledgments}

This work was supported in part by the National Key R\&D Project under Grant 2019YFB2005303, National Natural Science Foundation of China (no. 51805468), Natural Science Foundation of Jiangsu Province under Grant BK20180175, Key R\&D Program of Xuzhou of Jiangsu Province under Grant KC18096, and Natural Science Foundation of Hebei Province under Grant E2020203090.

\section{References}

[1] M. F. Shah, Z. Kausar, and M. M. Ahmad, "PID control and stability analysis of an ith leg of a six degree of freedom machining bed," Procedia Manufacturing, vol. 17, pp. 927934, 2018.

[2] L. Lyu, Z. Chen, and B. Yao, "Energy saving motion control of independent metering valves and pump combined hydraulic system," IEEE/ASME Transactions on Mechatronics, vol. 24, no. 5, pp. 1909-1920, 2019.

[3] L. Lyu, Z. Chen, and B. Yao, "Development of pump and valves combined hydraulic system for both high tracking precision and high energy efficiency," IEEE Transactions on Industrial Electronics, vol. 66, p. 1, 2018.

[4] L. Lyu, Z. Chen, and B. Yao, "Advanced valves and pump coordinated hydraulic control design to simultaneously achieve high accuracy and high efficiency," IEEE Transactions on Control Systems Technology, vol. 29, no. 99, pp. 1-13, 2020.

[5] A. Salimi Lafmejani, M. Tale Masouleh, and A. Kalhor, "Trajectory tracking control of a pneumatically actuated 6DOF Gough-Stewart parallel robot using BacksteppingSliding Mode controller and geometry-based quasi forward kinematic method," Robotics and Computer-Integrated Manufacturing, vol. 54, pp. 96-114, 2018.

[6] M. Wang, Y. Hu, Y. Sun et al., "An adjustable low-frequency vibration isolation Stewart platform based on electromagnetic negative stiffness," International Journal of Mechanical Sciences, vol. 181, Article ID 105714, 2020.

[7] Y.-S. Kim, H. Shi, N. Dagalakis, J. Marvel, and G. Cheok, "Design of a six-DOF motion tracking system based on a Stewart platform and ball-and-socket joints," Mechanism and Machine Theory, vol. 133, pp. 84-94, 2019.

[8] M. Russo and X. Dong, "A calibration procedure for reconfigurable Gough-Stewart manipulators," Mechanism and Machine Theory, vol. 152, Article ID 103920, 2020.

[9] Y. Ting, C.-C. Li, and T. V. Nguyen, "Composite controller design for a 6DOF Stewart nanoscale platform," Precision Engineering, vol. 37, no. 3, pp. 671-683, 2013.

[10] L. J. du Plessis and J. A. Snyman, "An optimally re-configurable planar Gough-Stewart machining platform," Mechanism and Machine Theory, vol. 41, no. 3, pp. 334-357, 2006.

[11] K. Herbuś and P. Ociepka, "Integration of the virtual model of a Stewart platform with the avatar of a vehicle in a virtual reality," IOP Conference Series: Materials Science and Engineering, vol. 145, Article ID 042018, 2016.

[12] D. Kuehn, M. Schilling, T. Stark, M. Zenzes, and F. Kirchner, "System design and testing of the hominid robot charlie," Journal of Field Robotics, vol. 34, no. 4, pp. 666-703, 2017.

[13] J. Yao, Y. Hou, H. Wang, T. Zhou, and Y. Zhao, "Spatially isotropic configuration of Stewart platform-based force sensor," Mechanism and Machine Theory, vol. 46, no. 2, pp. 142-155, 2011.

[14] M. Xia, Z. Xu, K. Han et al., "Dynamic disturbance force measurement platform for large moving device in spacecraft," Journal of Sound and Vibration, vol. 447, pp. 61-77, 2019.

[15] H. Yun, L. Liu, Q. Li, W. Li, and L. Tang, "Development of an isotropic Stewart platform for telescope secondary mirror," Mechanical Systems and Signal Processing, vol. 127, pp. 328344, 2019.

[16] Y. X. Su, B. Y. Duan, B. Peng, and R. D. Nan, "Singularity analysis of fine-tuning Stewart platform for large radio telescope using genetic algorithm," Mechatronics, vol. 13, no. 5, pp. 413-425, 2003.

[17] I. N. Egorov and D. S. Shevtsov, "Structure of system positionforce control of the drive Stewart platform," Procedia Computer Science, vol. 103, pp. 517-521, 2017.

[18] F. Yin, S. Nie, W. Hou, and S. Xiao, "Effect analysis of silencing grooves on pressure and vibration characteristics of seawater axial piston pump," Proceedings of the Institution of Mechanical Engineers-Part C: Journal of Mechanical Engineering Science, vol. 231, no. 8, pp. 1390-1409, 2017.

[19] D. E. Galván-Pozos and F. J. Ocampo-Torres, "Dynamic analysis of a six-degree of freedom wave energy converter based on the concept of the Stewart-Gough platform," Renewable Energy, vol. 146, pp. 1051-1061, 2019.

[20] Y. Fanglong, N. Songlin, H. Wei et al., "Effect analysis of silencing grooves on pressure and vibration characteristics of seawater axial piston pump," Proceedings of the Institution of Mechanical Engineers-Part C: Journal of Mechanical Engineering Science, vol. 231, no. 8, p. 1390, 2016.

[21] Y. Zhu, S. Tang, C. Wang, W. Jiang, X. Yuan, and Y. Lei, "Bifurcation characteristic research on the load vertical vibration of a hydraulic automatic gauge control system," Processes, vol. 7, no. 10, p. 718, 2019.

[22] S. Tang, S. Yuan, and Y. Zhu, "Deep learning-based intelligent fault diagnosis methods toward rotating machinery," IEEE Access, vol. 8, no. 1, pp. 9335-9346, 2020.

[23] Y. Zhu, S. Tang, L. Quan, W. Jiang, and L. Zhou, "Extraction method for signal effective component based on extremepoint symmetric mode decomposition and Kullback-Leibler 
divergence," Journal of the Brazilian Society of Mechanical Sciences and Engineering, vol. 41, no. 2, p. 100, 2019.

[24] Y. Zhu, S. Tang, C. Wang, W. Jiang, J. Zhao, and G. Li, "Absolute stability condition derivation for position closedloop system in hydraulic automatic gauge control," Processes, vol. 7, no. 10, p. 766, 2019.

[25] W. Li, E. Li, L. Ji, L. Zhou, W. Shi, and Y. Zhu, "Mechanism and propagation characteristics of rotating stall in a mixedflow pump,” Renewable Energy, vol. 153, p. 1, 2020.

[26] M. S. Alam, I. A. Yeasmin, and M. S. Ahamed, "Generalization of the modified Lindstedt-Poincare method for solving some strong nonlinear oscillators," Ain Shams Engineering Journal, vol. 10, no. 1, pp. 195-201, 2019.

[27] Y. Yan, W. Wang, and L. Zhang, "Applied multiscale method to analysis of nonlinear vibration for double-walled carbon nanotubes," Applied Mathematical Modelling, vol. 35, no. 5, pp. 2279-2289, 2011.

[28] F. Liang, A. Gao, X. F. Li et al., "Nonlinear parametric vibration of spinning pipes conveying fluid with varying spinning speed and flow velocity," Applied Mathematical Modelling, vol. 95, no. 8, p. 320, 2021.

[29] Y. Zhu, P. Qian, S. Tang, W. Jiang, W. Li, and J. Zhao, "Amplitude-frequency characteristics analysis for vertical vibration of hydraulic AGC system under nonlinear action," AIP Advances, vol. 9, no. 3, Article ID 035019, 2019.

[30] X. Q. He, M. Rafiee, S. Mareishi, and K. M. Liew, "Large amplitude vibration of fractionally damped viscoelastic CNTs/ fiber/polymer multiscale composite beams," Composite Structures, vol. 131, pp. 1111-1123, 2015.

[31] L.-Q. Chen, H. Ding, and C. W. Lim, "Principal parametric resonance of axially accelerating viscoelastic beams: multiscale Analysis and differential quadrature verification," Shock and Vibration, vol. 19, no. 4, 17 pages, Article ID 948459, 2012. 